\title{
Estimation of Forest Structure, Ground, and Canopy Layer Characteristics From Multibaseline Polarimetric Interferometric SAR Data
}

\author{
Maxim Neumann, Student Member, IEEE, Laurent Ferro-Famil, Member, IEEE, and \\ Andreas Reigber, Member, IEEE
}

\begin{abstract}
This paper concerns forest parameter retrieval from polarimetric interferometric synthetic aperture radar (PolInSAR) data considering two layers, one for the ground under the vegetation and one for the volumetric canopy. A model is designed to combine a physical model-based polarimetric decomposition with the random-volume-over-ground (RVoG) PolInSAR parameter inversion approach. The combination of a polarimetric scattering media model with a PolInSAR RVoG vertical structure model provides the possibility to separate the ground and the volume coherency matrices based on polarimetric signatures and interferometric coherence diversity. The proposed polarimetric decomposition characterizes volumetric media by the degree of polarization orientation randomness and by the particle scattering anisotropy. Using the full model enhances the estimation of the vertical forest structure parameters by enabling us to estimate the ground-to-volume ratio, the temporal decorrelation, and the differential extinction. For forest vegetation observed at $L$-band, this model accounts for the ground topography, forest and canopy layer heights, wave attenuation in the canopy, tree morphology in the form of the angular distribution and the effective shapes of the branches, and the contributions from the ground level consisting of surface scattering and double-bounce ground-trunk interactions, as well as volumetric understory scattering. The parameter estimation performance is evaluated on real airborne L-band SAR data of the Traunstein test site, acquired by the German Aerospace Center (DLR)'s E-SAR sensor in 2003, in both singleand multibaseline configurations. The retrieved forest height is compared with the ground-truth measurements, revealing, for the given test site, an average root-mean-square error (rmse) of about $5 \mathrm{~m}$ in the repeat-pass configuration. This implies an improvement in rmse by over $2 \mathrm{~m}$ in comparison to the pure coherence-based RVoG PolInSAR parameter inversion.
\end{abstract}

Index Terms-Forest structure, polarimetric decomposition, polarimetric synthetic aperture radar (SAR) interferometry, temporal decorrelation.

Manuscript received June 26, 2008; revised December 13, 2008 and May 11, 2009. First published October 30, 2009; current version published February 24, 2010.

M. Neumann and L. Ferro-Famil are with the SAPHIR Team, Institute of Electronics and Telecommunications of Rennes, University of Rennes 1, 35042 Rennes, France (e-mail: maxn@cs.tu-berlin.de; laurent.ferro-famil@ univ-rennes1.fr).

A. Reigber is with the SAR Technology Department, Microwaves and Radar Institute, German Aerospace Center (DLR), 82234 Wessling, Germany (e-mail: andreas.reigber@dlr.de).

Color versions of one or more of the figures in this paper are available online at http://ieeexplore.ieee.org.

Digital Object Identifier 10.1109/TGRS.2009.2031101

\section{INTRODUCTION}

$\mathbf{P}$ OLARIMETRIC synthetic aperture radar (SAR) interferometry (PolInSAR) provides strong means for vegetation parameter retrieval as it is sensitive to the vertical structure and physical characteristics of the scattering media.

On the one hand, polarimetry is sensitive to individual particle characteristics such as orientations, shapes, and permittivities, as well as ensemble average entropy [1]-[4]. Furthermore, model-based polarimetric decompositions permit separating main scattering contributions from vegetated areas which consist of surface, double-bounce, and volume scattering [5]-[10]. However, due to the limited number of observables (four polarimetric degrees of freedom), only very simple geophysical media models have been used for the ground-volume separation and parameter retrieval, limiting the applicability of model-based approaches. In particular, for surface and doublebounce scattering, only first-order models are used, whereas, for vegetation, only the randomly oriented volume is considered, with recent extension to a few discrete orientation states [9]. One topic of this paper is to provide a refined physical model for vegetation parameter retrieval which is not constrained by these limits.

On the other hand, interferometry is sensitive to topography, vertical structure, and density of the scattering media. The interferometric decorrelation in volumetric media has been recognized as an opportunity to measure vegetation depth and extinction [11]-[17]. The combination of interferometry with polarimetry enhances the estimation of the vertical structure by providing additional degrees of freedom. Several approaches have been developed using the polarization dependence of the interferometric coherence to evaluate the ground contribution and the linear ground-to-volume relationship, in order to estimate the vegetation height, the underneath ground topography, and the extinction in vegetation [18]-[23]. In particular, [20] presented an approach considering simple first-order ground responses and derived, in addition to the surface and volume coherences, also the double-bounce coherence and some combinations between these three components. In a subsequent approach [21], [22], a simplified model was presented consisting only of three structural degrees of freedom (ground phase, vegetation height, and extinction) and one additional degree of freedom for polarization diversity which steers the ground-to-volume ratio. However, this approach requires, at the one hand, a polarization with no ground contributions and, 
at the other hand, large coherence and ground contribution diversity in dependence of polarization. The first condition can only be fulfilled when all ground contributions have firstorder forms, as well. Particular difficulties to this polarimetric interferometric SAR (PolInSAR) coherence-based approach pose the temporal decorrelation, caused by temporal changes between the repeat-pass data acquisitions, and the inability to determine the ground-to-volume ratio. However, due to its simplicity and universality, the implementation of this approach is simple, and the computational costs are low. This led to successful validations of this coherence-based approach in various campaigns for different frequencies and configurations (e.g., [24]-[27]).

In this paper, we propose to combine a physical modelbased polarimetric decomposition with the PolInSAR randomvolume-over-ground ( $\mathrm{RVOG}$ ) coherence model for the vertical forest structure in order to enhance both application areas and to address some of the mentioned limitations. We present a general polarimetric interferometric model for vegetation parameter retrieval which offers a more flexible direct volume component and which does not restrict the ground contributions to simple first-order forms. The goal of this approach is to model separately the ground and volume contributions, in order to enhance the estimation of structural vegetation parameters, and to permit the retrieval of morphological vegetation parameters, as well as ground parameters under the vegetation for further analysis.

This model is intended for both single- (SB) and multibaseline $(\mathrm{MB})$ repeat-pass acquisitions, and the temporal change is taken into account with the goal to develop a parameter retrieval framework which is robust against temporal decorrelation. Initial results based on simulated data and neglecting temporal decorrelation were already presented in [28].

This paper consists of three main sections. In the next section, the forward model is presented based on the analysis of vegetation characteristics. We introduce the von Mises distribution as the expected unimodal circular polarization orientation angle distribution of the vegetation particles under the central limit theorem. A polarimetric coherency matrix form is derived for the volume component which depends only on the degree of orientation randomness and particle scattering anisotropy. Particle scattering anisotropy is a complex value describing the effective shape of the particles in the volume in dependence of their permittivities and tilt angles. Both orientation randomness and particle scattering anisotropy determine the morphology of the vegetation layer, as it is observable by radar. Using further means (some assumptions or a priori information), it is possible to infer further characteristics from the particle scattering anisotropy related to the distribution of tilt angles, the average permittivity, and particle shape ratio [29]. All ground contributions are combined into a single unconstrained component. This polarimetric model is complemented by an interferometric model consisting of system, temporal, and volumetric coherence constituents.

In Section III, the vegetation parameter inversion problem is discussed, and a parameter retrieval framework is presented.

In Section IV, the model and the parameter retrieval approaches are evaluated using real airborne SAR data, acquired
TABLE I

LIST OF SYMBOLS

\begin{tabular}{|c|c|c|}
\hline Symbol & Description & $\begin{array}{l}\text { First } \\
\text { appearance }\end{array}$ \\
\hline$g, v$ & indices for ground and volume layers & Fig. 1 \\
\hline$z_{0}$ & ground topography height & Fig. 1 \\
\hline$h_{v}$ & forest height & Fig. 1 \\
\hline$r_{h}$ & canopy-fill-factor & Fig. 1 \\
\hline$S$ & scattering matrix & (1) \\
\hline$S_{i j}$ & $\begin{array}{l}\text { scattering coefficient, the indices represent re- } \\
\text { ceive and transmit polarizations }\end{array}$ & (1) \\
\hline$h, v, a, b$ & $\begin{array}{l}\text { indices for polarizations (horizontal, vertical, } \\
\text { and eigenpolarizations) }\end{array}$ & (1) \\
\hline$\hat{\boldsymbol{p}}_{i}$ & polarization vectors & (1) \\
\hline $\boldsymbol{R}_{S(\psi)}$ & rotation matrix for $S$ & (2) \\
\hline$\psi$ & polarization orientation angle & (2) \\
\hline$\delta$ & particle scattering anisotropy & (3) \\
\hline$\widetilde{\psi}$ & mean polarization orientation & (6) \\
\hline$p_{\psi}$ & distribution of orientation angles (von Mises) & (6) \\
\hline$\kappa$ & degree of orientation concentration & (6) \\
\hline$I_{0}, I_{1}, I_{2}$ & $\begin{array}{l}\text { modified Bessel function of zeroth, first, and } \\
\text { second order }\end{array}$ & (6) \\
\hline$\tau$ & degree of orientation randomness & (7) \\
\hline $\boldsymbol{k}$ & scattering vector & (8) \\
\hline $\boldsymbol{T}$ & polarimetric coherency matrix & (8) \\
\hline $\mathrm{E}\{\cdot\}$ & expectation value operator & (8) \\
\hline$f_{g / v}$ & intensity normalization factors & (9) \\
\hline$\beta, \beta_{22}, \beta_{33}$ & ground coherency matrix parameterization & (10) \\
\hline $\boldsymbol{R}_{T}$ & rotation matrix for $\boldsymbol{T}$ & $(12)$ \\
\hline$g, g_{c}$ & auxiliary functions of $\tau$ for $\boldsymbol{T}_{v}$ & (14) \\
\hline$t_{i j}$ & i-j matrix elements of $\boldsymbol{T}_{v}$ & Fig. 4 \\
\hline $\boldsymbol{T}_{6}$ & PolInSAR coherency matrix & (17) \\
\hline$\Omega$ & PolInSAR cross-correlation coherency matrix & (17) \\
\hline$k_{z}$ & vertical wavenumber & (18) \\
\hline$k_{0}$ & wavenumber & (18) \\
\hline$B_{\perp}$ & perpendicular (spatial) baseline & $(18)$ \\
\hline$R_{0}$ & reference slant range distance & (18) \\
\hline$\theta_{0}$ & reference incidence angle & (18) \\
\hline $\boldsymbol{\omega}$ & polarization projection vector & (19) \\
\hline$\gamma$ & interferometric complex coherence & (19) \\
\hline$\phi$ & interferometric phase & (19) \\
\hline$\Gamma$ & PolInSAR coherence set & (20) \\
\hline$\phi_{0}$ & reference ground phase & $(23)$ \\
\hline$\gamma_{s y s}$ & system coherence & (23) \\
\hline$\gamma_{z}$ & volume coherence & (24) \\
\hline$\gamma_{r e s}$ & residual coherence & (24) \\
\hline$\gamma_{t e m p}$ & temporal coherence & after (24) \\
\hline$\sigma$ & mean extinction & $(25)$ \\
\hline$\Delta \sigma$ & differential extinction & after $(25)$ \\
\hline$c_{g}, c_{v}$ & normalized polarimetric power coefficients & (26) \\
\hline$d$ & set of data observables & Fig. 6 \\
\hline $\boldsymbol{m}$ & set of model parameters & Fig. 6 \\
\hline$G(\boldsymbol{m})$ & forward problem & Fig. 6 \\
\hline$G^{-1}(d)$ & inverse problem & Fig. 6 \\
\hline$L$ & number of looks & (30) \\
\hline$A, B, C, \ldots$ & general parameterization of $\boldsymbol{T}_{6}$ & (32) \\
\hline$c$ & set of constraints for parameter retrieval & (35) \\
\hline $\mathcal{L}$ & line representation & after $(35)$ \\
\hline$\Pi$ & PolInSAR contraction matrix & (36) \\
\hline$P_{g / v}$ & ground and volume component powers & (38) \\
\hline
\end{tabular}

by the German Aerospace Center (DLR)'s E-SAR system at L-band. An abundance of new parameters is estimated using the new model and the inversion framework. The most important estimated parameters, which characterize vegetation, are presented and discussed.

After the conclusion, two appendixes are added. The first details the polarimetric relationship of the derived parameter, particle scattering anisotropy, to the spheroidal particle model. The second appendix briefly outlines the general interferometric multilayer coherence model. For an overview, Table I 


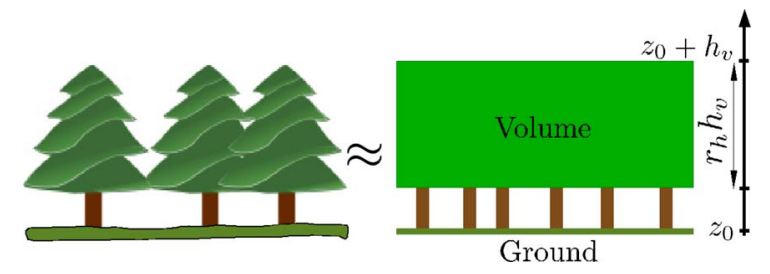

Fig. 1. Forest model parameterization: Ground $(g)$ and volume $(v)$ layers and the modeled vertical structure of the forest (ground topography $z_{0}$, forest height $h_{v}$, and the canopy fill factor $r_{h}$ ).

provides the list of symbols used in the main sections of this paper and their brief descriptions.

\section{MODEL}

The considered simplified forest model, consisting of a single homogeneous canopy layer above the ground, is shown in Fig. 1. The ground layer $(g)$ consists of all scattering contributions whose phase delays correspond to the absolute ground height $z_{0}$. This includes the direct surface scattering at the ground level, the double-bounce scattering between the ground and the tree trunks and branches, and the diffuse volume scattering from a thin layer of understory. The volume layer $(v)$, representing the forest canopy, extends over a fraction $r_{h}$ of the total forest height and is characterized by volumetric scattering. In this section, a general polarimetric and interferometric model is developed and presented, starting with the characterization of the vegetation.

\section{A. Vegetation Characterization}

A simplified volumetric vegetation layer can be characterized by a cloud of scattering particles whose electromagnetic properties are governed by the joint probability density function (pdf) of their positions, shapes, sizes, dielectric constants, and orientations. The single-particle scattering properties are assumed to be independent of position and orientation. The general scattering matrix in the lexicographic polarization basis $\hat{\boldsymbol{p}}_{h}, \hat{\boldsymbol{p}}_{v}$ is given by

$$
\boldsymbol{S}_{h v}=\left[\begin{array}{ll}
S_{h h} & S_{h v} \\
S_{v h} & S_{v v}
\end{array}\right]
$$

where $S_{r t}$ are the scattering coefficients and the indexes denote the combination of transmit $(t)$ and receive $(r)$ polarizations in terms of horizontal $(h)$ and vertical $(v)$ polarizations.

Under the hypothesis that particles have an axis of symmetry in the polarization plane, one may give the representative particle backscattering matrix in the eigenpolarization basis $\hat{\boldsymbol{p}}_{a}$, $\hat{\boldsymbol{p}}_{b}$ after rotation by the polarization angle $\psi$ as

$$
\begin{aligned}
\boldsymbol{S}_{a b} & =\boldsymbol{R}_{S(\psi)}^{T} \boldsymbol{S}_{h v} \boldsymbol{R}_{S(\psi)} \\
& =\left[\begin{array}{cc}
S_{a a} & 0 \\
0 & S_{b b}
\end{array}\right]=\frac{S_{a a}+S_{b b}}{2}\left[\begin{array}{cc}
1+\delta^{*} & 0 \\
0 & 1-\delta^{*}
\end{array}\right]
\end{aligned}
$$

where ${ }^{T}$ is the transposition operator, ${ }^{*}$ is the complex conjugate operator,

$$
\boldsymbol{R}_{S(\psi)}=\left[\begin{array}{cc}
\cos \psi & \sin \psi \\
-\sin \psi & \cos \psi
\end{array}\right]
$$

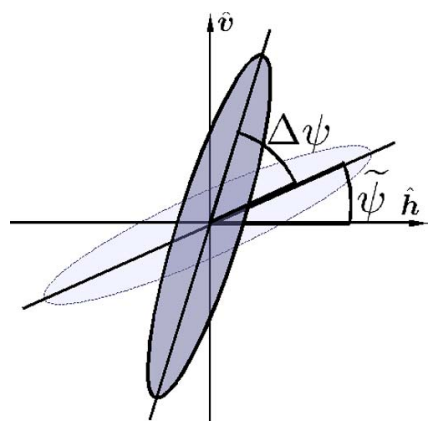

Fig. 2. Particle orientation in the polarization plane: Mean orientation $\widetilde{\psi}$ and the orientation of an individual particle $\psi=\widetilde{\psi}+\Delta \psi$.

is the rotation matrix for the polarization orientation angle change, and

$$
\delta=\left(\frac{S_{a a}-S_{b b}}{S_{a a}+S_{b b}}\right)^{*}
$$

is the particle scattering anisotropy, which describes the scattering properties of an average particle, as perceived by the radar, independently of polarization orientation and scattered power.

The particle scattering anisotropy characterizes the effective shape of the average particle in dependence of the particle and background permittivities and tilt angle distribution (see Appendix A). If the permittivities are significantly distinctive, one can make the following predictions about the effective particle shapes, assuming simple spheroidal particles: As $|\delta| \rightarrow 0$, the average effective particle shape approaches an isotropic sphere/disk, whereas, for $|\delta| \rightarrow 1$, the effective shape tends toward a dipole. If the phases of the scattering coefficients $S_{a a}$ and $S_{b b}$ are similar, then $\delta$ is a function only of their moduli. Then, in the line-of-sight (LOS) direction, the particle axis of symmetry tends to be horizontal if $\operatorname{Re} \delta>0$ and vertical if $\operatorname{Re} \delta<0$, with respect to the polarization basis of the particle scattering amplitude matrix. The interpretation of $\delta$ becomes more complex when the phases of $S_{a a}$ and $S_{b b}$ diverge.

The particle orientation angles $\psi$ in the polarization plane (with reference to the horizontal axis, as shown in Fig. 2) are assumed to follow a unimodal circular distribution $p_{\psi}(\psi)$ and to be independent from other vegetation characteristics. Under the central limit theorem condition, given a large number of scatterers, the orientations of these scatterers are normally distributed and follow the circular normal distribution (also known as the von Mises distribution) [30] which is the circular analog of the Gaussian distribution

$$
p_{\psi}(\psi \mid \widetilde{\psi}, \kappa)=\frac{e^{\kappa \cos (2(\psi-\widetilde{\psi}))}}{\pi I_{0}(\kappa)}, \quad \kappa \in[0, \infty]
$$

where $\kappa$ is the degree of concentration [analogous of the inverse of the standard deviation (SDEV)], $\widetilde{\psi} \in[-(\pi / 2),(\pi / 2)]$ is the mean orientation angle, and $I_{0}(\kappa)$ is the modified Bessel function of order 0 . For the sake of interpretation, the normalized degree of orientation randomness $\tau$ is introduced by

$$
\tau=I_{0}(\kappa) e^{-\kappa}, \quad \tau \in[0,1] .
$$




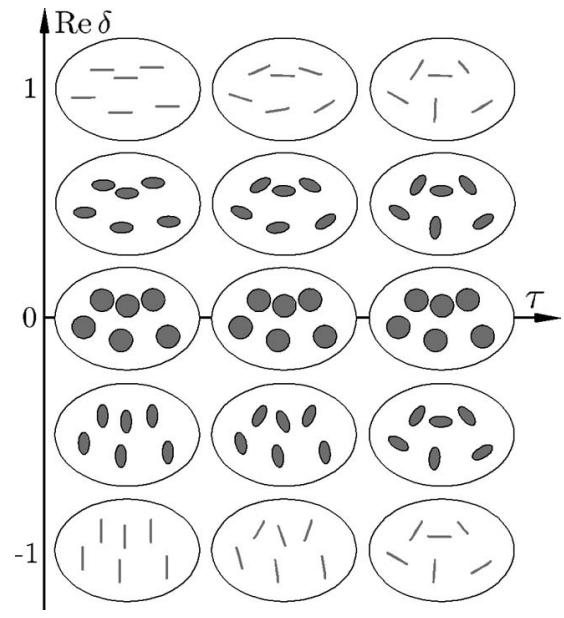

Fig. 3. Schematic representation of the effective shapes and orientations of particles in the LOS for different degrees of particle scattering anisotropy $\delta$ and orientation randomness $\tau$.

As $\tau \rightarrow 0$, the volume becomes strongly aligned in the preferred orientation direction, whereas, for $\tau \rightarrow 1$, the particle orientations become completely random. Fig. 3 schematically visualizes the volume particles in the direction of the LOS in dependence of the degrees of orientation randomness and particle scattering anisotropy [assuming that $\arg S_{a a} \approx \arg S_{b b}$ for $S_{a a}, S_{b b}$ from (3)]. Note that the degree of orientation randomness becomes meaningless for (effectively) isotropic scatterers $(\delta=0)$.

\section{B. Polarimetry}

Second-order scattering statistics for random media can be better represented in the Pauli matrix basis. Assuming reciprocity, the scattering vector and the coherency matrix are given in the Pauli basis by

$$
\boldsymbol{k}=\frac{1}{\sqrt{2}}\left[\begin{array}{c}
S_{h h}+S_{v v} \\
S_{h h}-S_{v v} \\
2 S_{h v}
\end{array}\right] \quad \boldsymbol{T}=\mathrm{E}\left\{\boldsymbol{k}^{\dagger}\right\}
$$

where ${ }^{\dagger}$ is the Hermitian operator and $\mathrm{E}\{\cdot\}$ is the expectation value operator.

In analogy to the Freeman-Durden model [7], the coherency matrix under the modeled assumptions can be decomposed into a ground layer and a volume layer

$$
\boldsymbol{T}=f_{g} \boldsymbol{T}_{g}+f_{v} \boldsymbol{T}_{v}
$$

where the individual coherency matrices are normalized with reference to their first elements so that the normalization factors $f_{g / v}$ are equal to the values $\mathrm{E}\left\{\left|S_{h h}+S_{v v}\right|^{2}\right\}$ of the individual contributions.

In forests, the ground layer contribution is composed of direct surface scattering from the ground, the double-bounce scattering between the surface and tree trunks and branches, and the diffuse volume scattering from understory. We explicitly do not consider a specific model for the contributions located at the ground level since an adequate model would be too complex. Instead, these contributions are combined to a single ground component. This combination does not restrict in any way the retrieval of other vegetation parameters in a repeat-pass acquisition configuration, as it will be considered in this paper.

In the general case, assuming only reciprocity and reflection symmetry [6], the ground coherency matrix can be represented in the ground eigenpolarizations by

$$
\boldsymbol{T}_{g}=\left[\begin{array}{ccc}
1 & \beta & 0 \\
\beta^{*} & \beta_{22} & 0 \\
0 & 0 & \beta_{33}
\end{array}\right]
$$

consisting of four polarimetric degrees of freedom $(\beta \in$ $\left.\mathbb{C}, \beta_{22}, \beta_{33} \in \mathbb{R}\right)$. With additional a priori knowledge or if a certain contribution dominates the ground response, one might apply further models to retrieve from the $\boldsymbol{T}_{g}$ characteristics related either to surface or double-bounce or understory scattering.

Using (2) and (8), the normalized first-order coherency matrix of a single particle is given by

$$
\hat{\boldsymbol{T}}_{v}(\psi)=\boldsymbol{R}_{T(2 \psi)}\left[\begin{array}{ccc}
1 & \delta & 0 \\
\delta^{*} & |\delta|^{2} & 0 \\
0 & 0 & 0
\end{array}\right] \boldsymbol{R}_{T(2 \psi)}^{\mathrm{T}}
$$

where

$$
\boldsymbol{R}_{T(2 \psi)}=\left[\begin{array}{ccc}
1 & 0 & 0 \\
0 & \cos 2 \psi & \sin 2 \psi \\
0 & -\sin 2 \psi & \cos 2 \psi
\end{array}\right]
$$

is the rotation matrix for coherency matrices. Since the particles are homogeneously distributed in the canopy layer, the volume component coherency matrix under the Born approximation can be obtained by the integration of these individual coherency matrices over the orientation angles [7]

$$
\begin{aligned}
\boldsymbol{T}_{v}= & \int_{-\pi / 2}^{\pi / 2} p_{\psi}(\psi) \hat{\boldsymbol{T}}_{v}(\psi) d \psi \\
= & \int_{-\pi / 2}^{\pi / 2} p_{\psi}(\psi) \\
& \times\left[\begin{array}{ccc}
1 & \delta \cos 2 \psi & -\delta \sin 2 \psi \\
\delta^{*} \cos 2 \psi & |\delta|^{2} \cos ^{2} 2 \psi & -|\delta|^{2} \cos 2 \psi \sin 2 \psi \\
-\delta^{*} \sin 2 \psi & -|\delta|^{2} \cos 2 \psi \sin 2 \psi & -|\delta|^{2} \sin ^{2} 2 \psi
\end{array}\right] d \psi
\end{aligned}
$$

where the integration is performed elementwise. The solution is, in general, not reflection symmetric. However, since $p_{\psi}(\psi)$ is a circular pdf, symmetric around the mean vegetation orientation $\widetilde{\psi}$, one can rotate the polarization orientation angle by $\widetilde{\psi}$ to obtain a reflection symmetric form

$\boldsymbol{T}_{v}=\boldsymbol{R}_{T(2 \widetilde{\psi})}\left[\begin{array}{ccc}1 & g_{c} \delta & 0 \\ g_{c} \delta^{*} & \frac{(1+g)}{2}|\delta|^{2} & 0 \\ 0 & 0 & \frac{(1-g)}{2}|\delta|^{2}\end{array}\right] \boldsymbol{R}_{T(2 \widetilde{\psi})}^{\mathrm{T}}$.

Some of the off-diagonal elements become zero since $p_{\psi}(\psi)$ is an even function with respect to $\widetilde{\psi}$. The values of $g$ and 


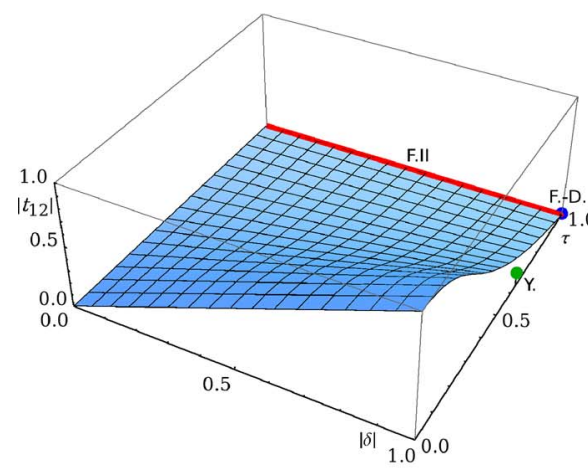

(a)

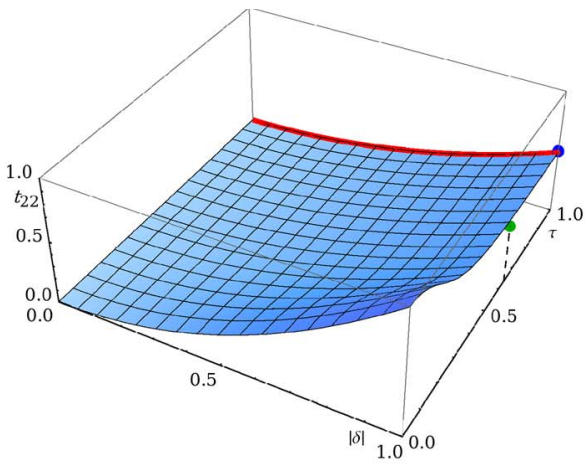

(b)

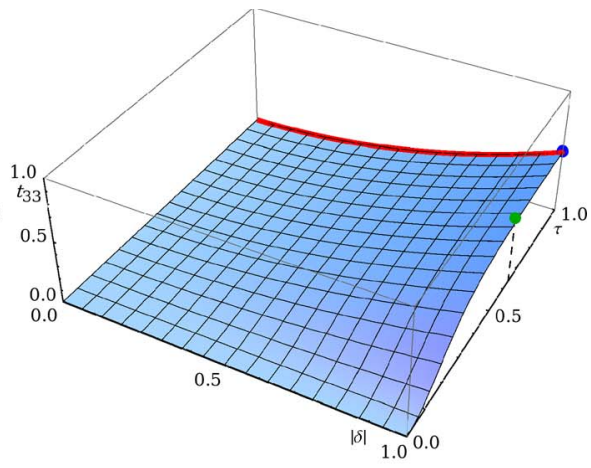

(c)

Fig. 4. Normalized volume coherency matrix elements $\left(\left|t_{12}\right|, t_{22}, t_{33}\right)$ as functions of the degree of orientation randomness $\tau$ and the particle scattering anisotropy $|\delta|$. The blue point, the red line, and the green point correspond to the parameter ranges of the Freeman-Durden, the Freeman II, and the Yamaguchi models, respectively. (a) $\left|t_{12}\right|=\left(\left|\mathrm{E}\left\{\left(S_{h h}+S_{v v}\right)\left(S_{h h}-S_{v v}\right)^{*}\right\}\right|\right) /\left(\mathrm{E}\left\{\left|S_{h h}+S_{v v}\right|^{2}\right\}\right)$. (b) $t_{22}=\left(\mathrm{E}\left\{\left|S_{h h}-S_{v v}\right|^{2}\right\}\right) /\left(\mathrm{E}\left\{\left|S_{h h}+S_{v v}\right|^{2}\right\}\right)$. (c) $t_{33}=\left(\mathrm{E}\left\{4\left|S_{h v}\right|^{2}\right\}\right) /\left(\mathrm{E}\left\{\left|S_{h h}+S_{v v}\right|^{2}\right\}\right)$.

$g_{c}$ are obtained using trigonometric and integral identities [30, Def. 9.6.19, p. 376]

$$
\begin{gathered}
g=\int_{-\pi / 2}^{\pi / 2} p_{\psi}(\psi) \cos 4 \psi d \psi=\frac{I_{2}(\kappa)}{I_{0}(\kappa)} \\
g_{c}=\int_{-\pi / 2}^{\pi / 2} p_{\psi}(\psi) \cos 2 \psi d \psi=\frac{I_{1}(\kappa)}{I_{0}(\kappa)}
\end{gathered}
$$

where $I_{n}$ are modified Bessel functions of $n$th order.

Equation (14) represents the most general form of direct volume backscattering from a simple homogeneous layer using a circular unimodal orientation angle distribution. In the reflection symmetric form (neglecting $\widetilde{\psi}$ ), the polarimetric properties of this component are determined by three real-valued parameters: the magnitude and phase of particle scattering anisotropy and the degree of orientation randomness. For an overview, the magnitudes of the normalized coherency matrix elements are plotted in Fig. 4 using the parameterization space of the degree of orientation randomness $\tau \in[0,1]$ and the particle scattering anisotropy magnitude $|\delta| \in[0,1]$. For comparison, the parameter ranges for the volume components of the Freeman-Durden decomposition [7], the Freeman II decomposition [8], and the additional orientation-sensitive Yamaguchi decomposition [9] are represented by the blue point, the red curve, and the green point, respectively.

To rotate the whole coherency matrix $\boldsymbol{T}$ into a reflection symmetric form requires the normal vector of the ground terrain to be in the plane of the volume eigenpolarizations so that both surface and volume share a common eigenpolarization basis (same azimuthal orientation). If this assumption is valid, one might also be able to estimate the terrain slopes under vegetation based on polarimetry only, as it is done for bare surfaces, for instance, in [4].

\section{Polarimetric Interferometry}

The expectation value of the SB PolInSAR coherency matrix [18] under polarimetric stationarity [31] and reciprocity conditions is given by

$$
\boldsymbol{T}_{6}=\mathrm{E}\left\{\boldsymbol{k}_{6} \boldsymbol{k}_{6}^{\dagger}\right\}=\left[\begin{array}{cc}
\boldsymbol{T} & \boldsymbol{\Omega} \\
\boldsymbol{\Omega}^{\dagger} & \boldsymbol{T}
\end{array}\right], \quad \boldsymbol{k}_{6}=\left[\begin{array}{l}
\boldsymbol{k}_{1} \\
\boldsymbol{k}_{2}
\end{array}\right]
$$

where $\boldsymbol{k}_{1}$ and $\boldsymbol{k}_{2}$ are the scattering vectors describing the same scene but from slightly different incidence angles and possibly different times. The wave interferometric properties are characterized (after appropriate preprocessing) by the vertical wavenumber

$$
k_{z}=2 k_{0} \frac{B_{\perp}}{R_{0} \sin \theta_{0}}
$$

where $k_{0}, B_{\perp}, R_{0}$, and $\theta_{0}$ are the wavenumber, the effective (perpendicular) baseline, the slant range distance, and the incidence angle, respectively. The main PolInSAR observable is the complex coherence, which can be computed by

$$
\gamma(\boldsymbol{\omega})=|\gamma| e^{i \phi}=\frac{\boldsymbol{\omega}^{\dagger} \boldsymbol{\Omega} \boldsymbol{\omega}}{\boldsymbol{\omega}^{\dagger} \boldsymbol{T} \boldsymbol{\omega}}
$$

where $\boldsymbol{\omega}$ is a polarization projection vector determined by the choice of transmit and receive polarizations.

Considering all possible combinations of transmit and receive polarizations provides a range of coherences, called the coherence set

$$
\Gamma=\left\{\gamma(\boldsymbol{\omega}) \mid \boldsymbol{\omega} \in \mathbb{C}^{3}\right\} .
$$

In analogy to the polarimetric model of $\boldsymbol{T}$ in (9), the interferometric behavior of the main scattering contributions from a layer of random volume vegetation over the ground can be modeled by a linear combination of polarimetric interferometric cross-correlation matrices

$$
\boldsymbol{\Omega}=f_{g} \boldsymbol{\Omega}_{g}+f_{v} \boldsymbol{\Omega}_{v}
$$

Under the assumption of polarization independence of all decorrelation sources, (21) can be expressed by

$$
\boldsymbol{\Omega}=f_{g} \boldsymbol{T}_{g} \gamma_{g}+f_{v} \boldsymbol{T}_{v} \gamma_{v}
$$

where $\gamma_{g}$ and $\gamma_{v}$ are the interferometric complex coherence terms associated with the ground and volume layers. 


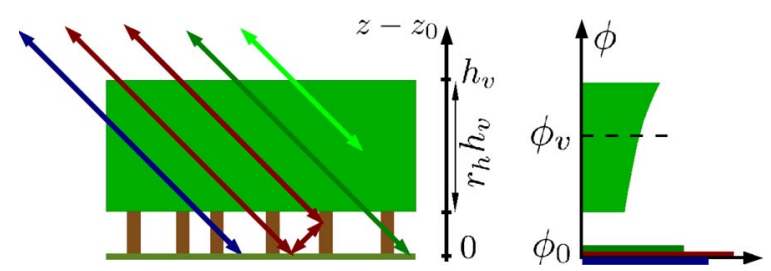

Fig. 5. Model for the interferometric phase profile due to the vertical distribution of scattering mechanism types. The surface, double-bounce, and short understory cause similar interferometric phases at the ground level. The volume layer introduces a phase offset and an additional decorrelation due to phase variation. The wave attenuation in the volume layer causes less scattering at lower heights inside the layer.

The interferometric criterion for separating the ground from the volume is based on the interferometric phase and coherence. As outlined in Fig. 5, in the repeat-pass configuration, the surface scattering of the ground, the double-bounce scattering between the ground and the tree trunks and branches, and the scattering from very short understory cause similar interferometric ground-locked phases and degrees of decorrelation, making the aggregation of these terms possible. The vertical distribution of scatterers in the volume layer causes the corresponding interferometric phases to be distributed in a certain range, which introduces a phase offset and a volumetric source of decorrelation.

As shown in Appendix B, after conventional PolInSAR data processing (calibration, coregistration, spectral range filtering, flat earth removal, etc.), the ground coherence term can be expressed by

$$
\gamma_{g}=e^{i \phi_{0}} \gamma_{\mathrm{sys}}
$$

where $\phi_{0}$ is the reference ground phase and $\gamma_{\text {sys }}$ is the system decorrelation which represents the acquisition system and data processing errors, including the thermal noise.

The volume layer coherence is given by

$$
\gamma_{v}=e^{i \phi_{0}} \gamma_{\mathrm{res}} \gamma_{z}
$$

where $\gamma_{z}$ is the volume decorrelation caused by the vertical forest structure and $\gamma_{\mathrm{res}}$ is the residual decorrelation term which combines all other decorrelation sources, including system decorrelation and temporal decorrelation $\gamma_{\text {temp }}$. In this paper, $\gamma_{\text {sys }}$ and $\gamma_{\text {res }}$ are approximated by polarization-independent real-valued terms which only degrade the coherence magnitude without affecting the interferometric phase.

The volume coherence $\gamma_{z}$ for vegetation with the canopy layer extending to a fraction $r_{h}$ of the total vegetation height $h_{v}$, as shown in Fig. 1, is given by

$$
\gamma_{z}=e^{i k_{z}\left(1-r_{h}\right) h_{v}} \frac{\int_{0}^{r_{h} h_{v}} e^{\left(\frac{2 \sigma}{\cos \theta_{0}}+i k_{z}\right) z} d z}{\int_{0}^{r_{h} h_{v}} e^{\frac{2 \sigma}{\cos \theta_{0}} z} d z}
$$

where the extinction coefficient $\sigma$ accounts for the mean attenuation of the electromagnetic waves in the volume layer.

In general, in the presence of orientation effects in the volume, extinction becomes polarization dependent [19], [20], [32]. In this case, the volume coherence at the eigenpolarizations will be given by replacing the mean extinction value $\sigma$ in (25) by $\sigma \pm \Delta \sigma . \Delta \sigma$ is the differential extinction and is primarily related to the degree of orientation randomness $\tau$, as it tends toward zero for $\tau \rightarrow 1$ and toward $\sigma$ for $\tau \rightarrow 0$.

However, for the scenario considered in this paper, that is, forest parameter retrieval at L-band in repeat-pass acquisitions, the degree of orientation randomness is very high, the mean extinction is low, and, hence, interferometry is hardly sensitive to extinction and even less to the polarization-dependent variation of extinction. Therefore, to keep the model and the parameter retrieval framework simple, extinction is approximated by a polarization-independent scalar value. However, the full polarimetric interferometric model with extinction and refractivity differences in the volume layer is derived in [32], and estimates of the differential extinction $\Delta \sigma$ based on polarimetry are presented in Section IV.

Alternatively, (22) can be represented by a coherence model

$\gamma_{(\boldsymbol{\omega})}=c_{g(\boldsymbol{\omega})} \gamma_{g}+c_{v(\boldsymbol{\omega})} \gamma_{v}=c_{g(\boldsymbol{\omega})} \gamma_{g}+\left(1-c_{g(\boldsymbol{\omega})}\right) \gamma_{v}$

where the $c_{i}$ coefficients are the normalized polarimetric power coefficients

$$
c_{i(\boldsymbol{\omega})}=f_{i} \frac{\boldsymbol{\omega}^{\dagger} \boldsymbol{T}_{i} \boldsymbol{\omega}}{\boldsymbol{\omega}^{\dagger} \boldsymbol{T} \boldsymbol{\omega}}, \quad i \in\{g, v\} \quad \forall \boldsymbol{\omega}: \sum_{i} c_{i(\boldsymbol{\omega})}=1 .
$$

This coherence model formulation resembles the ones in [12], [14], [20], [21], and [33], but, combined with a polarimetric decomposition, the full PolInSAR model becomes more efficient for vegetation parameter retrieval by having more degrees of freedom and using synergy effects. For instance, the polarimetric scattering model determines the ground-to-volume ratio, which is an important parameter in the RVoG model, enabling the determination of $\gamma_{v}$ without ambiguity.

\section{Multiple Baselines}

The SB PolInSAR model can readily be scaled to multiple baselines, given $n$ data acquisitions

$\boldsymbol{T}_{3 n}=\mathrm{E}\left\{\boldsymbol{k}_{3 n} \boldsymbol{k}_{3 n}^{\dagger}\right\}=\left[\begin{array}{ccc}\boldsymbol{T} & \ldots & \boldsymbol{\Omega}_{1 n} \\ \vdots & \ddots & \vdots \\ \boldsymbol{\Omega}_{1 n}^{\dagger} & \ldots & \boldsymbol{T}\end{array}\right], \quad \boldsymbol{k}_{3 n}=\left[\begin{array}{c}\boldsymbol{k}_{1} \\ \vdots \\ \boldsymbol{k}_{n}\end{array}\right]$

where every baseline $i-j(i, j \in[1, n])$ and every crosscorrelation matrix $\boldsymbol{\Omega}_{i j}$ are characterized by a distinctive vertical wavenumber $k_{z i j}$ and a temporal delay between the acquisitions. The acquisition of more observables can enhance the retrieval of modeled parameters. However, with every baseline, two parameters are added to the model: the reference ground phase $\phi_{0_{i j}}$ and the residual decorrelation of the volume $\gamma_{\mathrm{res}_{i j}}$, which are both, in general, unknown a priori.

In a similar manner, multiangular multialtitude multifrequency models can be constructed, where, in dependence of the acquisition properties, the polarimetric coherency matrices $\boldsymbol{T}$ will be different. 


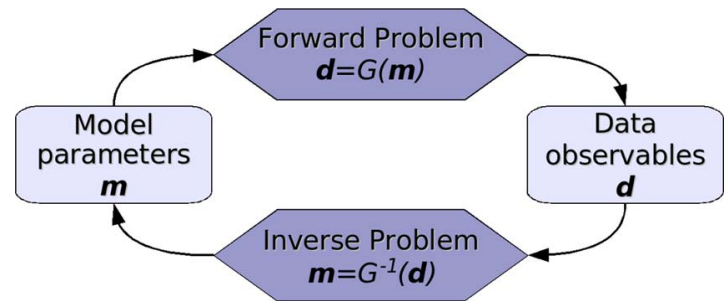

Fig. 6. General framework of a forward and an inverse problem.

\section{Vegetation PARAmeter Retrieval}

Vegetation parameter retrieval can be understood as an inverse problem [34] as outlined in Fig. 6.

\section{A. Data Observables}

The data observables are represented by the estimates of the polarimetric coherency matrix and the cross-correlation matrix

$$
\boldsymbol{d}=\{\hat{\boldsymbol{T}}, \hat{\boldsymbol{\Omega}}\} .
$$

The initial estimate of $\hat{\boldsymbol{T}}_{6}^{\prime}$ is obtained by averaging (also called multilooking) of data samples using $L$ looks

$$
\hat{\boldsymbol{T}}_{6}^{\prime}=\left[\begin{array}{ll}
\hat{\boldsymbol{T}}_{11} & \hat{\boldsymbol{\Omega}}_{12} \\
\hat{\boldsymbol{\Omega}}_{12}^{\dagger} & \hat{\boldsymbol{T}}_{22}
\end{array}\right]=\left\langle\boldsymbol{k}_{6} \boldsymbol{k}_{6}^{\dagger}\right\rangle_{L}=\frac{1}{L} \sum_{i=1}^{L} \boldsymbol{k}_{6_{i}} \boldsymbol{k}_{6_{i}}^{\dagger} .
$$

A maximum likelihood estimator for the polarimetric stationary form of the polarimetric coherency matrix is given by [31]

$$
\hat{\boldsymbol{T}}=\frac{\hat{\boldsymbol{T}}_{11}+\hat{\boldsymbol{T}}_{22}}{2} .
$$

Most vegetated areas exhibit reflection symmetry with respect to the vertical incidence plane, and the eigenpolarizations are given by the $\mathrm{H}-\mathrm{V}$ polarization basis. However, in the presence of azimuth slopes of the terrain, the polarization orientation angle might be different and need to be estimated, for instance, from a digital elevation model or directly from polarimetry [4].

The final estimator of the SB PolInSAR coherency matrix with reciprocity, reflection symmetry, and polarimetric stationarity assumptions has the form

$$
\hat{\boldsymbol{T}}_{6}=\left[\begin{array}{cc}
\hat{\boldsymbol{T}} & \hat{\boldsymbol{\Omega}} \\
\hat{\boldsymbol{\Omega}}^{\dagger} & \hat{\boldsymbol{T}}
\end{array}\right]\left\{\begin{array}{c}
\hat{\boldsymbol{T}}=\left[\begin{array}{ccc}
A & D+i E & 0 \\
D-i E & B & 0 \\
0 & 0 & C
\end{array}\right] \\
\hat{\boldsymbol{\Omega}}=\left[\begin{array}{ccc}
F+i G & L+i M & 0 \\
N+i O & H+i I & 0 \\
0 & 0 & J+i K
\end{array}\right]
\end{array}\right.
$$

where the parameterization variables $(A, B, C, \ldots)$ represent the individual observables obtained after multilooking. Therefore, the set of independent observables for an SB coherency matrix under the named assumptions contains 15 real-valued elements [35]: one for the backscattered power, four for polarimetry, and ten for polarimetric interferometry. These are the ten additional parameters from interferometry, which enable us to construct more complex but still invertible polarimetric models for the ground and vegetation components. With the help of interferometry, we seek to separate two coherency matrices which should be representable for the ground and the volume contributions. At the same time, the coherences, which separate these two components, permit estimating the structural vegetation parameters and the degree of temporal decorrelation.

\section{B. Model Parameters}

With reference to Fig. 6, the set of modeled parameters for an SB scenario is given by

$$
\begin{array}{r}
\boldsymbol{m}=\left\{\widetilde{\psi}, f_{g}, \operatorname{Re} \beta, \operatorname{Im} \beta, \beta_{22}, \beta_{33}, f_{v}, \operatorname{Re} \delta, \operatorname{Im} \delta, \tau,\right. \\
\left.h_{v}, r_{h}, \sigma, \gamma_{\mathrm{sys}}, \gamma_{\mathrm{res}}, \phi_{0}\right\} .
\end{array}
$$

These are 16 real-valued model parameters, as presented in the previous section, which determine the modeled PolInSAR coherency matrix $\boldsymbol{T}_{6}$. One degree of freedom $\widetilde{\psi}$ is used to rotate the coherency matrix into a reflection symmetric form, which leaves 15 degrees of freedom. Of the remaining parameters, the first nine determine the polarimetric properties, whereas the last six determine the interferometric properties of the model.

The critical point of the whole parameter retrieval framework is to estimate the interferometric structural parameters correctly. With the knowledge of the acquisition system properties, $\gamma_{\mathrm{sys}}$ will be assumed to be known a priori. Hence, $\phi_{0}$ is estimable from $\gamma_{g}$.

Furthermore, $\gamma_{v}$ provides only 2 degrees of freedom $\left(\left|\gamma_{v}\right|, \arg \gamma_{v}\right)$ but determines four parameters, namely, $h_{v}, r_{h}$, $\sigma$, and $\gamma_{\text {res }}$. In addition, the magnitude of $\gamma_{v}$ is perturbed by $\gamma_{\text {res }}$ which is dominated by the unconstrained temporal decorrelation $\gamma_{\text {temp }}$. One approach to estimate the structural parameters from $\gamma_{v}$ is to use multiple baselines. With every distinctive baseline, we obtain the independent estimates of $\gamma_{g_{i}}$ and $\gamma_{v_{i}}$, in dependence of $k_{z}$, and two additional degrees of freedom $\phi_{0_{i}}$ and $\gamma_{\mathrm{res}_{i}} . \phi_{0_{i}}$ can be estimated with $\gamma_{g_{i}}$, whereas $\left|\gamma_{\mathrm{res}_{i}}\right|$ perturbs $\left|\gamma_{v_{i}}\right|$. Therefore, to estimate the three parameters $h_{v}$, $r_{h}$, and $\sigma$ requires the usage of at least three baselines with distinctive vertical wavenumbers.

\section{Parameter Retrieval Framework}

The direct model $G(\boldsymbol{m})$, which is related to the forward problem in Fig. 6, is given by

$$
\boldsymbol{d}=G(\boldsymbol{m}) \Longleftrightarrow\left\{\begin{aligned}
\boldsymbol{T}= & f_{g} \boldsymbol{T}_{g}\left(\beta, \beta_{22}, \beta_{33}\right)+f_{v} \boldsymbol{T}_{v}(\delta, \tau) \\
\boldsymbol{\Omega}= & f_{g} \boldsymbol{T}_{g}\left(\beta, \beta_{22}, \beta_{33}\right) \gamma_{g}\left(\phi_{0}, \gamma_{\mathrm{sys}}\right) \\
& +f_{v} \boldsymbol{T}_{v}(\delta, \tau) \gamma_{v}\left(\phi_{0}, \gamma_{\mathrm{res}}, h_{v}, r_{h}, \sigma\right)
\end{aligned}\right.
$$

where the individual terms are given by the equations in Section II.

The inverse model can be represented as a minimization problem, which is subject to a set of constraints $c$

$$
\boldsymbol{m}=G^{-1}(\boldsymbol{d}) \Longleftrightarrow \boldsymbol{m}=\underset{\boldsymbol{m} \mid \boldsymbol{c}}{\arg \min }\|\boldsymbol{d}-G(\boldsymbol{m})\| .
$$

The norm $\|\cdot\|$ is given by the $L_{2}$ vector norm of the argument (root-mean-square misfit minimization). $G^{-1}$ is a 


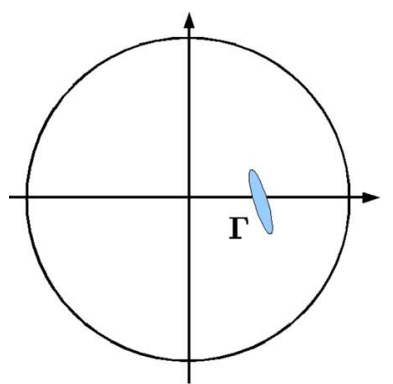

(a)

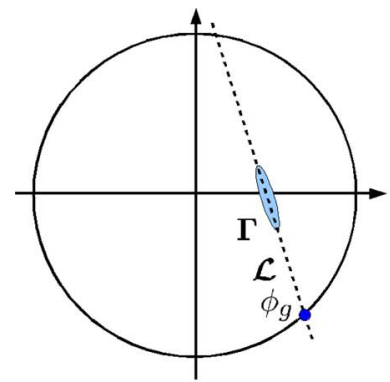

(b)

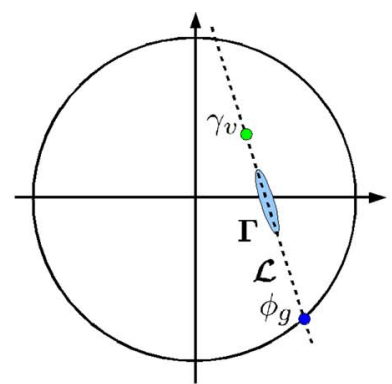

(c)

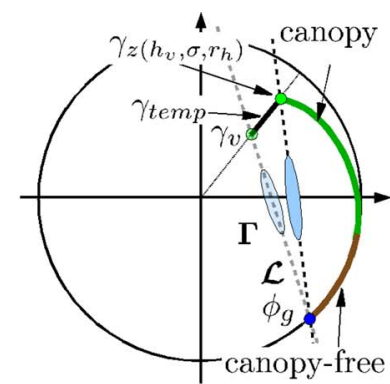

(d)

Fig. 7. Basic workflow of the parameter retrieval inside the complex coherence plane (neglecting $\gamma_{\mathrm{sys}}$ for the sake of simplicity of representation). (a) Initial coherence set. (b) Estimation of the linear structure and the ground phase. (c) Estimation of the ground-to-volume ratio and the volume layer coherence. (d) Estimation of the temporal decorrelation and the structural parameters from the true volume coherence.

multidimensional nonlinear optimization problem with local minima and possibly nonphysical solutions. The set of constraints $c$ is provided to enforce physically reasonable solutions. It proved useful to include into $\boldsymbol{d}$, next to the elements of the PolInSAR coherency matrix, several coherence values with distinctive polarizations to enhance the sensitivity of the parameter retrieval method to structural parameters.

While the problem formulation is straightforward, finding a solution is an art in itself. Using brute-force optimization might lead to undesirable results, and a physically refined approach is recommended. A possible multistage parameter retrieval framework for an MB data set, as outlined in Fig. 7, is presented in the following.

\section{Initial Processing:}

i) SAR image preprocessing: data generation, calibration, coregistration, range spectral filtering, flat earth removal, topography removal, and multilooking operation;

ii) estimation of the reflection symmetric and polarimetric stationary form [31];

iii) estimation of thermal decorrelation and possibly other system decorrelation sources, as, for example, the coherence bias [36] in the case of low number of looks.

\section{Parameter Retrieval:}

1) Determine the linear structures of the PolInSAR coherence sets.

Independently, for every baseline:

a) Fit a line $\mathcal{L}_{i}$ through the PolInSAR coherence set $\Gamma$, in analogy to the three-stage inversion process [22].

b) Determine the ground phase $\phi_{0_{i}}$.

2) Determine the degree of orientation randomness in the data.

For all baselines, simultaneously:

a) Find $\delta^{\prime}, \tau^{\prime}, f_{g}^{\prime}, f_{v}^{\prime}, \beta^{\prime}, \beta_{22}^{\prime}$, and $\beta_{33}^{\prime}$ which minimize (35), neglecting for the moment the structural parameters and only enforcing all $\gamma_{g_{i}}, \gamma_{v_{i}}$ to be on the lines $\mathcal{L}_{i}$.

b) Keep only $\tau=\tau^{\prime}$ for future computation.

3) Determine the structural parameters and temporal decorrelation, together with other remaining parameters.

a) Find common $h_{v}, \sigma$, and $r_{h}$, as well as $\delta, f_{g}, f_{v}$, $\beta, \beta_{22}, \beta_{33}$, and the baseline-dependent $\gamma_{\mathrm{res}_{i}}$ which approximate the linear structure of the coherences and the polarimetric coherency matrix.
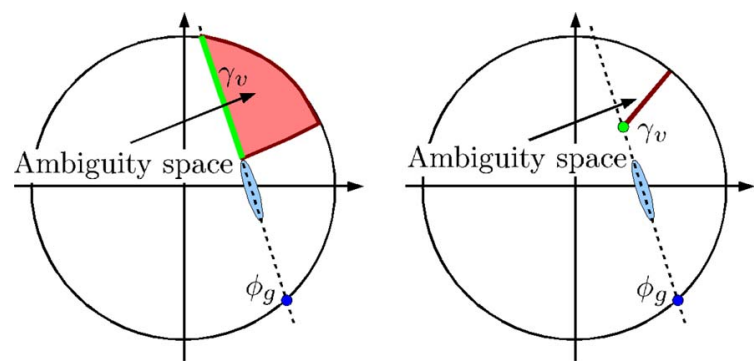

Fig. 8. Ambiguity space of the (left) coherence-based PolInSAR RVoG and the (right) proposed polarimetric model-based PolInSAR parameter inversion approaches.

4) If the retrieved parameters are physically not meaningful, either restart using different initialization or mark this pixel as noninverted and continue.

For step 1a), we propose to use the eigenvalues of the contraction matrix $\Pi$

$$
\Pi=\boldsymbol{T}^{-\frac{1}{2}} \boldsymbol{\Omega} \boldsymbol{T}^{-\frac{1}{2}}
$$

to estimate the linear structure of the coherences. The line function is estimated in polar coordinates.

Step 1b) is very important as errors in determining all ground phases correctly will result in erroneous parameter retrieval. The used criteria for identifying the ground phase [22] are the following: 1) polarimetric ordering of coherences and 2) maximal phase distance between the ground and the volume coherence. Other criteria are possible.

Step 2) has been introduced to make the procedure more robust. Theoretically, after step 2), the structural parameters can directly be retrieved from $\gamma_{v_{i}}$. However, the full parameter inversion in step 3) (except $\tau$ ) provides the possibility of further fine adjustment using the information from all baselines.

The Nelder-Mead simplex method [37] is used for the optimization problems in steps 2a) and $3 a$ ), which does not guarantee the optimal solution but which, even with repeated trials, is computationally effective. The usage of a more sophisticated optimization method, like simulated annealing or genetic algorithms, would provide better results, but the computation cost will be increased in this case.

Fig. 8 visualizes the advantage to combine the common coherence-based RVoG PolInSAR parameter inversion method with a polarimetric model. It enables us to estimate the exact 


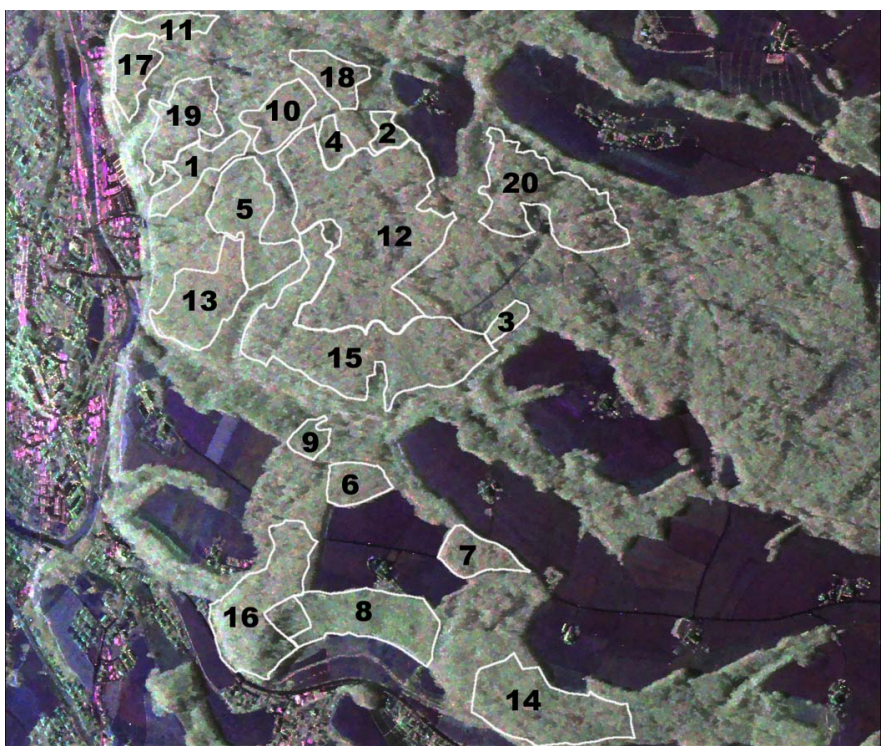

Fig. 9. Test site Traunstein: L-band SAR image in the Pauli basis.

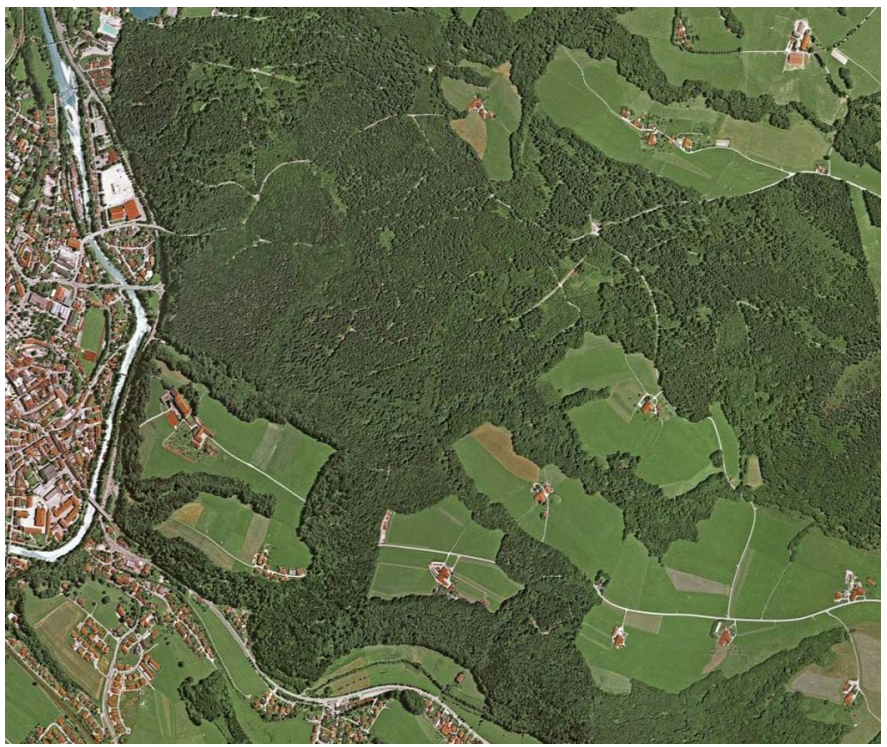

Fig. 10. Test site Traunstein: Optical image. Source: Google Earth

value of $\gamma_{v}$ by evaluating the ground-to-volume ratio and reducing with it the coherence ambiguity space. Using multiple baselines, the estimation of the temporal decorrelation and the true volume coherence $\gamma_{z}$ becomes feasible.

\section{EXPERIMENTAL RESUlts}

\section{A. Data Presentation}

The application of the developed parameter retrieval method is conducted on real SAR data from a mountainous temperate forested region in the south of Germany, near the city of Traunstein. The scene is presented in Figs. 9 and 10, showing L-band SAR intensity and optical images. The image rangeazimuth extension is $1414 \times 2379$ pixels, with a resolution of $1.5 \mathrm{~m}$ in range and $0.95 \mathrm{~m}$ in azimuth. The topography of the forest stands is relatively flat with only a few steep

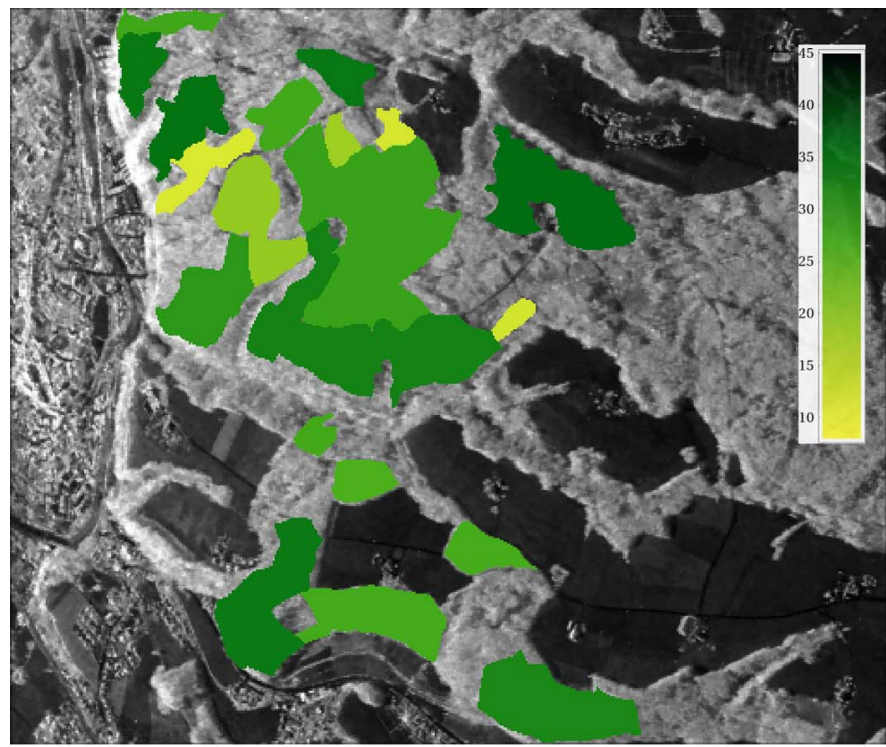

(a)

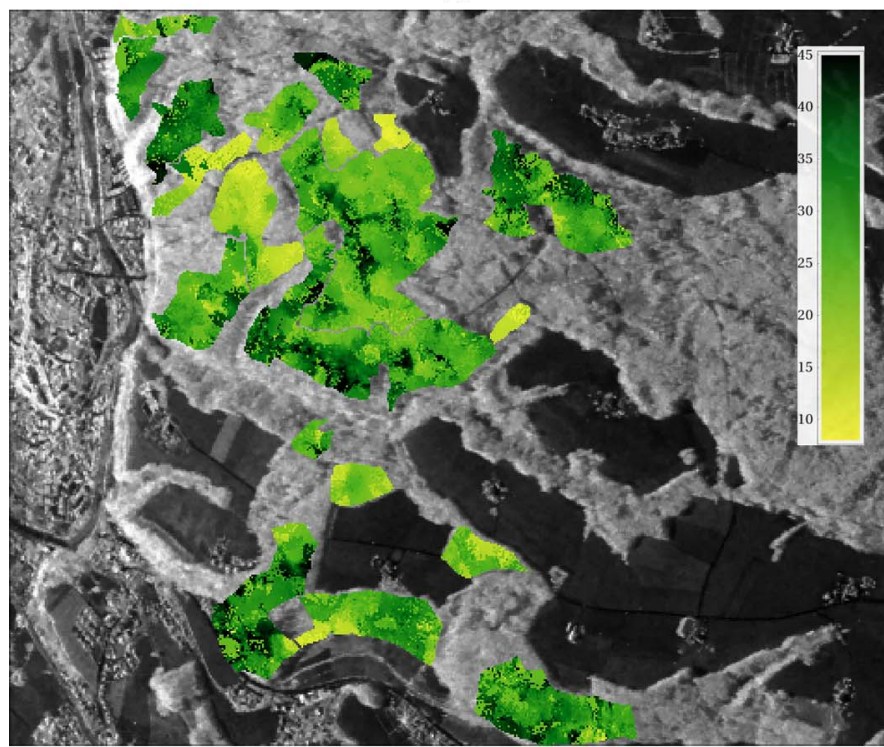

(b)

Fig. 11. Forest height estimation. Ground-truth and estimated height images. (a) Ground-truth forest height. (b) Estimated forest height.

slopes. The ground-truth data are available for 20 validation stands covering 123 ha, which are delimited in Fig. 9, and their main characteristics are presented in Table II. These individual stands were delineated in order to achieve high homogeneity in terms of tree species, height, biomass, and growth stadium [38]. Quantitative ground-truth information has been generated from the forest inventory in 1998 based on a $100 \times 100 \mathrm{~m}(1 \mathrm{ha})$ grid. It should be noted that the selection of homogeneous areas is subjective, and the provided quantitative parameters are naturally subject to variance. The growth stadium of the forest stands is related to age and current condition of the trees, dividing the stands into the classes of growth, mature, and regenerating. The forest stands can, in general, be characterized as mixed forests. Table II presents the dominant tree species on the order of dominance. The species shortcuts have the following meanings: $\mathrm{Fi}=$ north spruce, $\mathrm{Ki}=\mathrm{Scots}$ pine, $\mathrm{Ta}=$ white fir, 
TABLE II

Main Ground-Truth CHARACTERISTICS OF THE 20 Forest StANDS, (From Left to Right) Stand Number, GRowth Stadium ( $\mathrm{G}=$ Growth, $\mathrm{M}=$ MATURE, $\mathrm{R}=$ REgEnERATING)

Dominant Tree SPecies Type (c = Coniferous, $d=$ Deciduous $)$ Dominant Species, Average Height, Height Variation, AND SAR INCIDENCE ANGLE

\begin{tabular}{c|c|c|c|c|c|c}
\hline Stand & Stad. & T & Species & Avg. height & \pm height & $\theta_{0}$ \\
\hline \hline 1 & G & c & Fi-Ta-Bu & $12.46 \mathrm{~m}$ & $\pm 2.3 \mathrm{~m}$ & $37.3^{\circ}$ \\
2 & G & c & Fi & $13.00 \mathrm{~m}$ & $\pm 0 \mathrm{~m}$ & $44.8^{\circ}$ \\
3 & G & d & Ah-Bu-Fi & $13.05 \mathrm{~m}$ & $\pm 0 \mathrm{~m}$ & $48.4^{\circ}$ \\
4 & R & c & Fi-Bu & $18.66 \mathrm{~m}$ & $\pm 3.6 \mathrm{~m}$ & $42.9^{\circ}$ \\
5 & G & c & Fi-Ta-Bu & $19.68 \mathrm{~m}$ & $\pm 8.6 \mathrm{~m}$ & $39.7^{\circ}$ \\
6 & G & c & Fi-Bu-Ei & $26.3 \mathrm{~m}$ & $\pm 1.5 \mathrm{~m}$ & $43.7^{\circ}$ \\
7 & G & c & Fi-Bu-Ei & $26.93 \mathrm{~m}$ & $\pm 2.3 \mathrm{~m}$ & $47.5^{\circ}$ \\
8 & G & d & Bu-Ah-Es & $27.20 \mathrm{~m}$ & $\pm 3.1 \mathrm{~m}$ & $44.3^{\circ}$ \\
9 & G & d & Bu-Fi-La & $27.32 \mathrm{~m}$ & $\pm 0 \mathrm{~m}$ & $42.1^{\circ}$ \\
10 & M & c & Fi-Bu-La & $27.43 \mathrm{~m}$ & $\pm 2.2 \mathrm{~m}$ & $40.9^{\circ}$ \\
11 & G & c & Fi-Bu-La & $27.62 \mathrm{~m}$ & $\pm 2.2 \mathrm{~m}$ & $35.5^{\circ}$ \\
12 & M & c & Fi-Bu-Ta & $28.43 \mathrm{~m}$ & $\pm 1.6 \mathrm{~m}$ & $44.0^{\circ}$ \\
13 & M & c & Fi-Bi-Bu & $30.13 \mathrm{~m}$ & $\pm 2.7 \mathrm{~m}$ & $37.6^{\circ}$ \\
14 & M & c & Fi-Bu-Es & $32.49 \mathrm{~m}$ & $\pm 1.6 \mathrm{~m}$ & $49.3^{\circ}$ \\
15 & R & c & Fi-Bu-Ta & $33.14 \mathrm{~m}$ & $\pm 2.7 \mathrm{~m}$ & $43.7^{\circ}$ \\
16 & R & c & Fi-Bu-Ah & $34.34 \mathrm{~m}$ & $\pm 1.8 \mathrm{~m}$ & $40.2^{\circ}$ \\
17 & M & c & Fi-Bu-Ei & $34.59 \mathrm{~m}$ & $\pm 2.5 \mathrm{~m}$ & $34.0^{\circ}$ \\
18 & R & c & Fi-Ta-Ki & $34.66 \mathrm{~m}$ & $\pm 1.4 \mathrm{~m}$ & $42.9^{\circ}$ \\
19 & R & c & Fi-Ta-Bu & $35.23 \mathrm{~m}$ & $\pm 1.2 \mathrm{~m}$ & $36.5^{\circ}$ \\
20 & R & c & Fi-Bu & $36.10 \mathrm{~m}$ & $\pm 1.8 \mathrm{~m}$ & $49.4^{\circ}$ \\
\hline
\end{tabular}

TABLE III

DATA SET Characteristics: ACQUisition TIME, NOMINAL BASELINE, AND VERTICAL WAVENUMBER RANGE

\begin{tabular}{c|c|c|c}
\hline Track & Acquisition time & Nominal baseline & $k_{z}$ range \\
\hline \hline 1 & $11.11 .2003,09: 00$ & master & master \\
2 & $11.11 .2003,08: 50$ & $5 \mathrm{~m}$ & $0.062-0.112$ \\
3 & $11.11 .2003,08: 40$ & $10 \mathrm{~m}$ & $0.097-0.225$ \\
4 & $11.11 .2003,08: 01$ & $0 \mathrm{~m}$ & $0.012-0.088$ \\
\hline
\end{tabular}

TABLE IV

CHARACTERISTICS OF THE SAR IMAGERY

\begin{tabular}{l|l}
\hline \hline Wavelength & $23 \mathrm{~cm}$ \\
Polarizations & $\mathrm{HH}, \mathrm{HV}, \mathrm{VV}, \mathrm{VH}$ \\
Bandwidth & $100 \mathrm{MHz}$ \\
Altitude (above ground) & $3 \mathrm{~km}$ \\
Slant range resolution & $1.5 \mathrm{~m}$ \\
Azimuth resolution & $95 \mathrm{~cm}$ \\
Incidence angles & $25^{\circ}-56^{\circ}$ \\
\hline
\end{tabular}

$\mathrm{La}=$ Eur. larch, $\mathrm{Bu}=$ Eur. beech $, \mathrm{Es}=\mathrm{ash}, \mathrm{Ah}=$ maple, $\mathrm{Ei}=$ oak, and $\mathrm{Bi}=$ birch. Of the 20 stands, 17 are dominantly coniferous, and only three stands are dominated by deciduous trees, as denoted in the type column. The average tree heights of the evaluation stands range between 12.46 and $36.10 \mathrm{~m}$ with spatial variations inside the stands of up to $8.6 \mathrm{~m}$. For a better overview, the rows in the table are colored in dependence of the tree species type (blue for dominantly deciduous and green for coniferous species) and the growth stadium (the green color lightness relates to the growth stadium of the coniferous forest stands).

The fully polarimetric and interferometric data at L-band have been acquired by the DLR's E-SAR sensor in 2003 in a repeat-pass configuration. The acquisition times and nominal baselines of the four data sets used in this study are presented in Table III. Furthermore, the acquisition system characteristics are shown in Table IV.
TABLE V

A Priori And Optimization Constraints

\begin{tabular}{c|c}
\hline A priori constraints & Optimization constraints \\
\hline \hline & $0 \leq|\delta| \leq 1.5$ \\
$\gamma_{s y s}=0.96$ & $0 \mathrm{~dB} / \mathrm{m} \leq \sigma \leq 0.4 \mathrm{~dB} / \mathrm{m}$ \\
& $0.4 \leq r_{h} \leq 1$ \\
& $0.25 \leq \gamma_{r e s} \leq 1$ \\
& $5 \mathrm{~m} \leq h_{v} \leq 45 \mathrm{~m}$ \\
\hline
\end{tabular}

\section{B. Inversion Procedure Setup}

To guarantee good estimates of the covariance matrices and the coherences, the first series of tests is conducted using 1800 looks, which, with the given slant range and azimuth resolutions, correspond to an area of 0.25 ha (slant range geometry). 1800 looks can be obtained by a $15 \times 15$ boxcar averaging of the data after an $8 \times$ multilooking in the azimuth. Later on, the results will be presented for 450,900 , and 1350 looks $(1 / 16,1 / 8$, and 3/16 ha). Three baselines are used: $1-2,1-3$, and $1-4$. The system coherence, which includes thermal, miscoregistration, miscalibration, and other decorrelation sources, as well as possible temporal decorrelation of the ground contributions, is set a priori to 0.96 independently of the polarization.

Furthermore, the constraints, used to regularize the parameter retrieval method to deliver physically reasonable solutions and to avoid local minima, are presented in Table V.

\section{Results}

In this section, we present the first parameter retrieval results on real SAR data using the developed model which provide an abundance of new retrievable parameters. From all the retrieved parameters, only the forest height can be related to the groundtruth data for evaluation and validation purposes. To evaluate the parameter retrieval performance for other parameters, two other criteria are considered, namely, the SDEV and the distinctiveness. The SDEV of the parameter, which should be small inside a forest stand, can be related to the accuracy of the parameter estimation. The distinctiveness of the parameters between different forest stands, which should be high, is a measure of identification and classification of forest species types.

1) Vertical Forest Structure Estimation: Fig. 11(a) and (b) shows the ground-truth forest heights and the estimated heights. Fig. 12 shows the corresponding scatter plot of the heights. These images indicate already that the heights are estimated close to the ground truths. However, in some stands, the forest heights vary continuously, indicating certain inhomogeneities inside the stands.

Fig. 13 shows the forest heights and the individual canopy layer depths for the 20 evaluation stands. The red line in this plot represents the ground-truth height. The green line represents the estimated forest heights, whereas the lengths of the error bars are given by the SDEVs. The brown line delimits the canopy layer from the noncanopy layer above the ground and represents the canopy fill factor, normalized to the total vegetation height. In average, the forest height is underestimated by $1.5 \mathrm{~m}$, the average root-mean-square error (rmse) is $4.97 \mathrm{~m}$, and the average SDEV of the height estimation is $4.33 \mathrm{~m}$. 


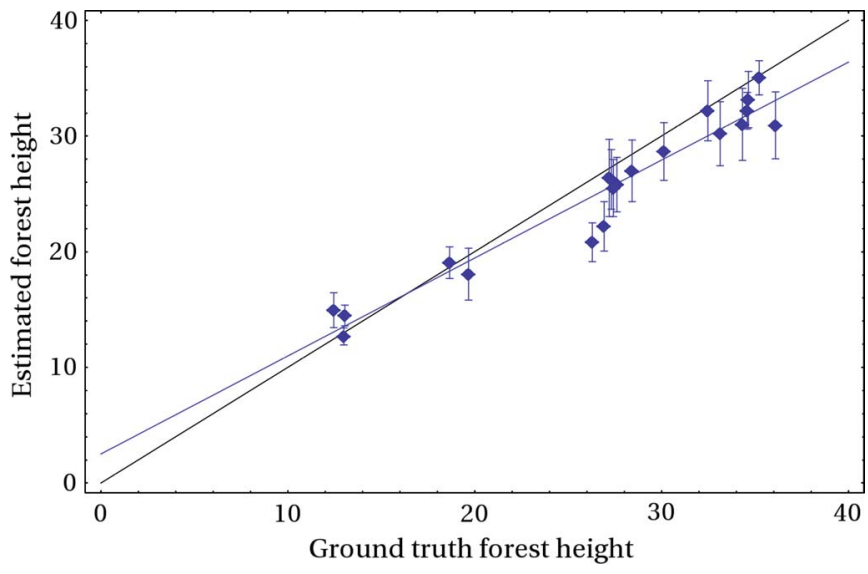

Fig. 12. Scatter plot of the estimated forest heights over the ground-truth forest heights. The error bars denote the estimated forest height SDEV.

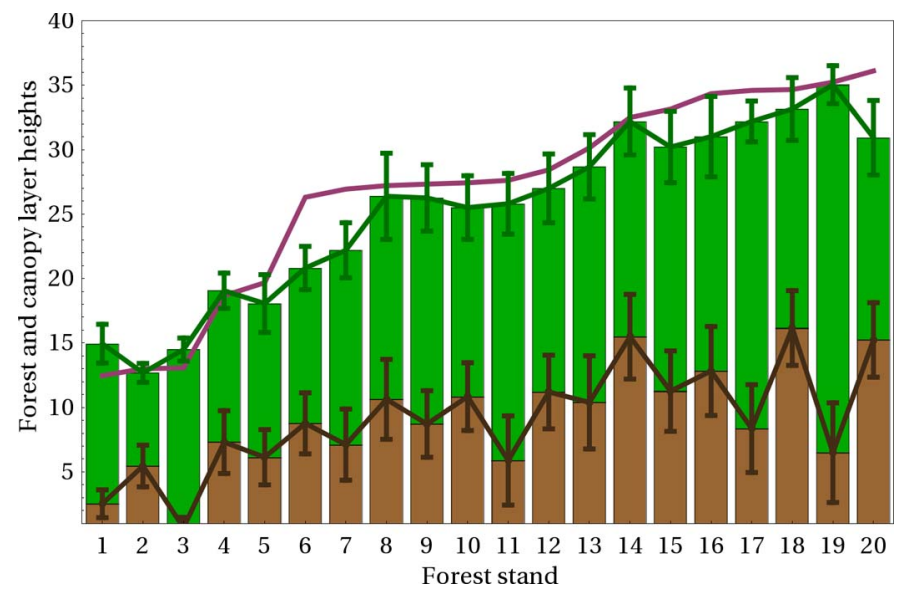

Fig. 13. Forest height estimation. Canopy layer and total vegetation depths. (Red) Ground-truth forest height. (Green) Estimated forest height. (Brown) Bottom height of the canopy layer. (Error bars) Related SDEV.

It is interesting to see that the lowest height estimation error corresponds to the only forest stand which is dominated by a single tree species (forest stand 2: bias $-32 \mathrm{~cm}$; rmse $1.5 \mathrm{~m}$ ).

The highest error corresponds to stand number 20 . The forest heights seem to be underestimated by over $5 \mathrm{~m}$ with an rmse of $7.75 \mathrm{~m}$. After the examination of the characteristics of this stand, we could identify two possible reasons for the errors. First, the ellipticity of the coherence sets is very high, and the coherence sets are often shrunk to small circles, making it difficult to estimate the linearity of the coherences which introduces severe errors in the estimation of ground phases. Second, looking at the optical image (Fig. 10), one can see that there are several clear-cuts and roads inside the limits of this forest stand. It is very likely that, between the forest inventory in 1998, on which base the evaluation stands have been defined, and the date of radar data acquisition in 2003, there have been significant changes in the spatial structure of this stand. Under this evidence, it seems likely that the "groundtruth" measurements do not correspond to the true forest height of this stand, which appears to be quite heterogeneous. This emphasizes again the capability of radar remote sensing for quantitative vegetation monitoring and parameter retrieval on spatial and temporal scales not achievable by other means.
TABLE VI

Height Estimation Statistics For THE MB AND SB CASES

\begin{tabular}{lc|ccc}
\hline & Baselines & Bias & RMSE & SDEV \\
\hline \hline MB: & $1-2,1-3,1-4$ & -1.51 & 4.97 & 4.33 \\
SB: & $1-2$ & 2.18 & 5.03 & 4.09 \\
\hline
\end{tabular}

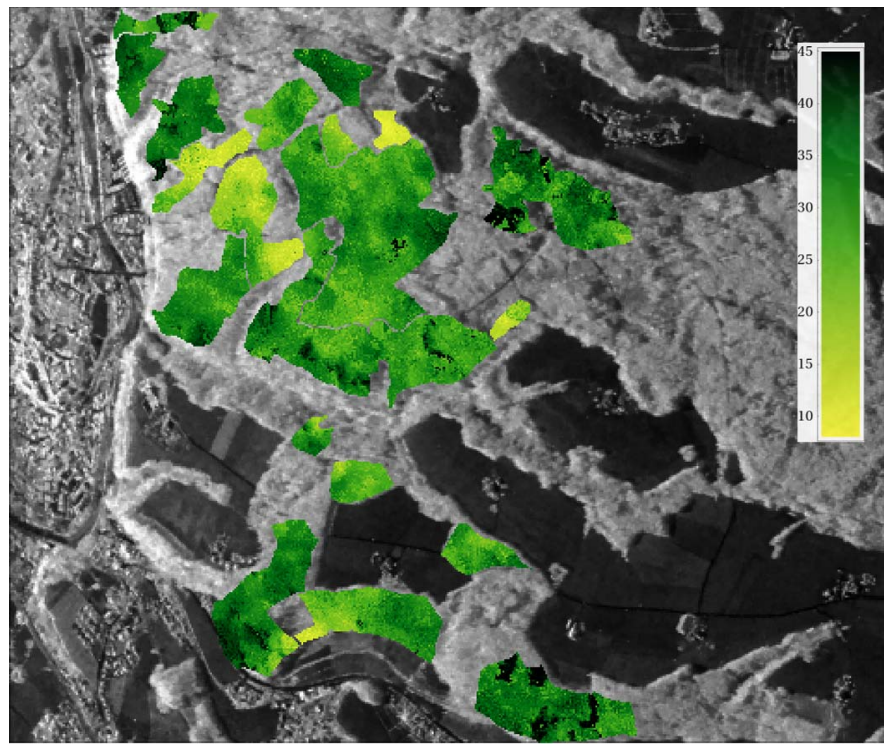

Fig. 14. Estimated forest height using an SB: 1-2.

2) SB Parameter Estimation: In this section, the forest height estimation performance using an SB is examined. Reducing the number of baselines and, with it, the $k_{z}$ diversity, one increases the ambiguity in the estimation of the structural parameters. In particular, when an SB is used, it is not possible anymore to estimate $\sigma$ and $r_{h}$, next to the residual decorrelation. However, limiting the range of $\sigma$ and $r_{h}$, one can still obtain reasonable estimates for the total forest height, given an accurate estimation of the ground-to-volume ratio, as shown in Table VI and Fig. 14 which present the parameter estimation results using an SB (1-2, 5-m nominal baseline, 10-min temporal separation).

In comparison of the SB with the three-baseline forest height estimation results, one can observe that the SB results are more biased and overestimated. This emphasized the difficulty to estimate the degree of temporal decorrelation using an SB. The temporal decorrelation gets underestimated, which results in apparently lower volume coherence values and higher forest height estimates. However, we were still able to obtain very robust and relatively accurate forest height estimates with a mean SDEV of $4 \mathrm{~m}$.

The same SB data set, using the 5-m baseline 1-2, has been analyzed for forest height and biomass estimation in [38] using the pure coherence-based RVoG model and inversion approach [22], [21]. A comparison of the results is not possible since the exact processing chain is not known, and a lower number of looks has been used. The SDEV of the estimated forest height was reported to be between 5 and $15 \mathrm{~m}$ for the individual forest stands, and the mean SDEV was more than $10 \mathrm{~m}$. In our own implementation of the pure coherence-based RVoG parameter inversion using the same data set and the data 


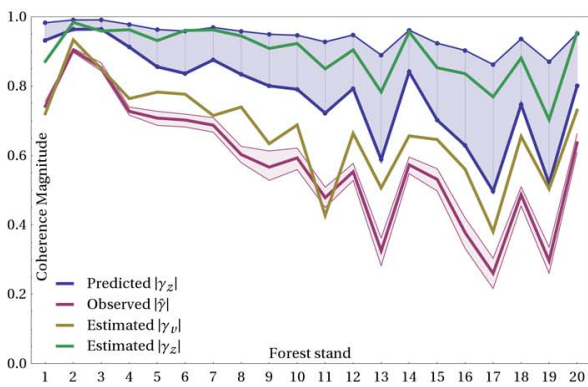

(a)

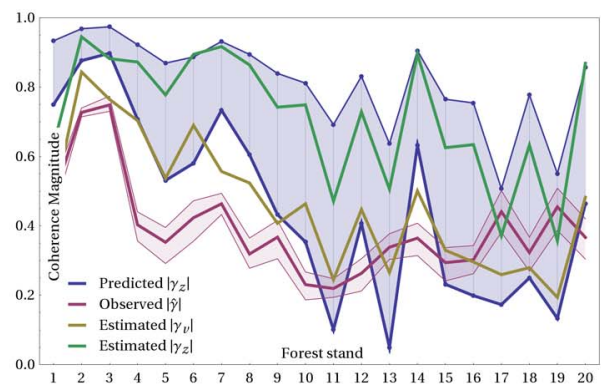

(b)

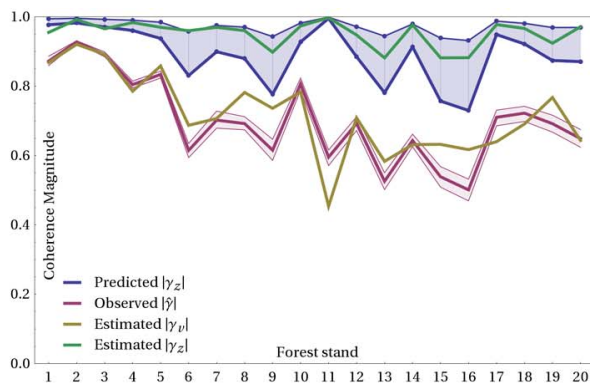

(c)

Fig. 15. Predicted, observed, and estimated coherence magnitude for all baselines. (a) Baseline 1. (b) Baseline 2. (c) Baseline 3.

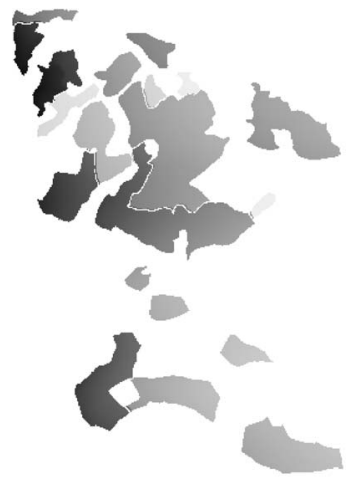

(a)

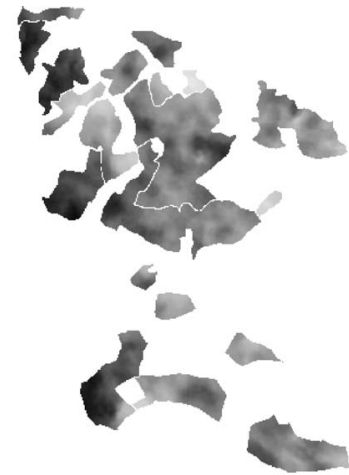

(b)

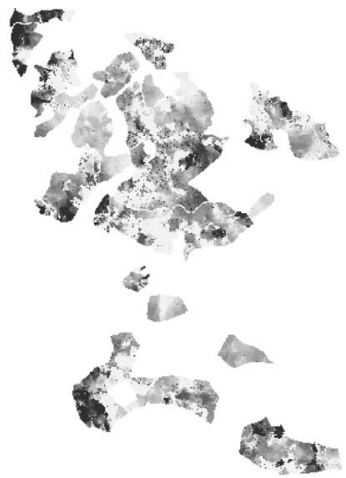

(c)

Fig. 16. Coherence magnitude images for baseline 1. Predicted $\left|\gamma_{z}\right|$ based on the given ground-truth forest heights and assuming a canopy fill factor $r_{h}=0.8$ and extinction $\sigma=0.01 \mathrm{~dB} / \mathrm{m}$, observed coherence in the data $|\gamma|$ (averaged over all the Pauli-basis polarizations), and the estimated residual coherence $\gamma_{\text {res }}$. (a) Predicted $\left|\gamma_{z}\right|\left(r_{h}=0.8, \sigma=0.01 \mathrm{~dB} / \mathrm{m}\right)$. (b) Observed $|\gamma|$. (c) Estimated residual coherence $\gamma_{\mathrm{res}}$.

processing as presented in this paper, we were able to obtain an average rmse of $7.2 \mathrm{~m}$. Therefore, using the full polarimetric and interferometric model and inversion approach increased the rmse accuracy of forest height estimation for the given data set by more than $2 \mathrm{~m}$, next to providing additional indicators for forest structure and further polarimetric characteristics of the ground and canopy layers.

3) Residual and Temporal Decorrelation Estimation: Prior to discussing the estimation of temporal decorrelation, it is worth to examine the expected and the observed coherence magnitude ranges over the different baselines. In Fig. 15, the ranges of the observed, predicted, and estimated coherence magnitudes are plotted over individual forest stands. The range of the observed coherences after preprocessing is shown in red. The predicted range of the volume coherence, based on the ground-truth forest heights from Table II and the acquisition system geometry, is shown in blue. The bottom thick blue line represents the maximal possible volumetric decorrelation assuming that the homogeneous canopy occupies the whole forest height range $\left(r_{h}=1\right)$ and neglecting any extinction $(\sigma=0)$. The top thin blue line represents a less decorrelating forest structure with $r_{h}=0.5$ and $\sigma=0.2 \mathrm{~dB} / \mathrm{m}$.

It can be seen that the observed coherence follows the trend of the predicted coherence range most of the time, except for the high forest stands in the largest baseline (baseline 2). However, there is still a big offset between the observed and the predicted coherences, which is due to various causes. First of all, the shown observed coherence combines the contributions from the ground and the volume. The evaluation of the ground-to-volume ratio will provide the estimate of the volume component coherence $\left|\gamma_{v}\right|$ represented by the yellow line. Next, the remaining offset between $\left|\gamma_{v}\right|$ and the estimated volumetric coherence $\left|\gamma_{z}\right|$, represented by the green line, is due to residual decorrelation sources. These residual components are dominated by the temporal and thermal decorrelation, but they are as well affected by other nonmodeled contributions due to, for instance, nonhomogeneous vertical structure, the polarization dependence of the individual coherence terms, the spatial heterogeneity inside of the forest stands, and residual errors after spectral range filtering, coregistration, etc.

As an example, the images of the predicted and observed coherence magnitudes for the first baseline are shown in Fig. 16(a) and (b). The estimated residual coherence is presented in Fig. 16(c). The average estimates of $\gamma_{\text {res }}$ for all baselines are shown in Fig. 17. The three baselines (data sets 1-2, 1-3, and 1-4) have nominal perpendicular baselines of 5, 10, and $0 \mathrm{~m}$ and temporal separations of 10,20 , and $60 \mathrm{~min}$. Over these short temporal periods, the number of minutes between the acquisitions is not authoritative. The temporal decorrelation of the volume at these scales is mostly caused by wind which is nonstationary, neither temporally nor spatially. Because of this behavior, our attempts to model the temporal decorrelation, e.g., by Brownian motion [39], [40], failed. It does not mean that it is not possible, but, given the few temporal samples 


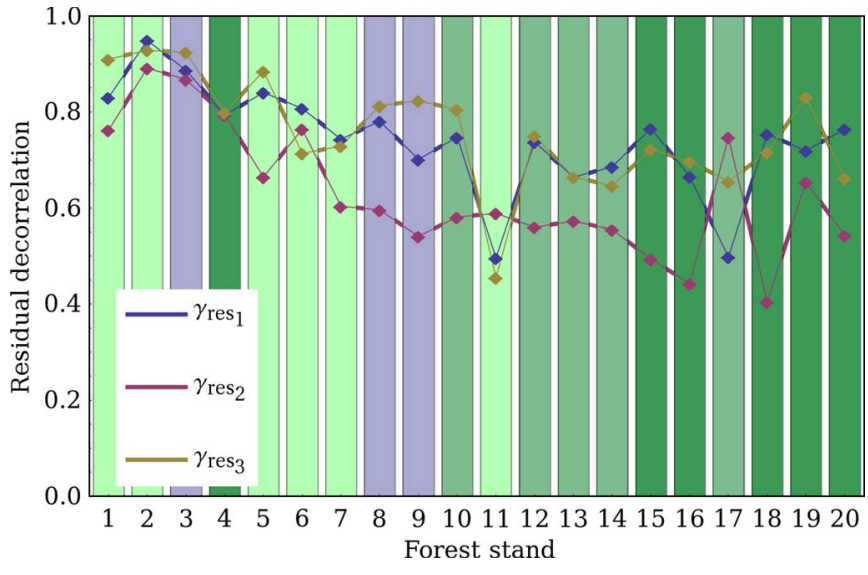

Fig. 17. Residual decorrelation for all the evaluation stands.

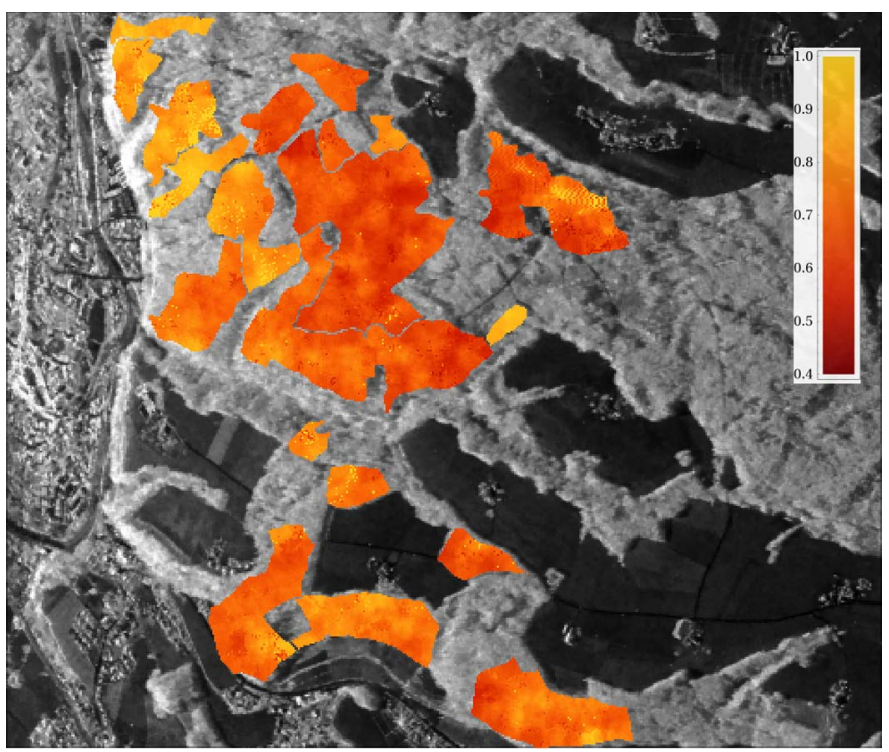

Fig. 18. Image of the degree of orientation randomness $\tau$.

and the high-resolution requirements, the models could not be applied successfully. These effects have to be studied using a larger space of spatial and temporal samples [26]. Therefore, we limited our study to the estimation of the temporal (i.e., residual) decorrelation in order to remove it and to estimate reliably the vegetation parameters.

As it can be seen, in average, the residual decorrelation is between 0.5 and 0.95 . We can observe that the estimated residual decorrelation is slightly correlated with the forest height and with the spatial baseline over some individual forest stands. The reason for this behavior is unknown; it might be related to contributions from other modeled or nonmodeled coherence constituents or to strong wind conditions at the time of the acquisition. In particular, we use a very simple model assuming a homogeneous layer with a single polarization-independent extinction value. The temporal behavior of the forest and the dependence of temporal decorrelation on tree morphology and branching structure have still to be studied.

4) Orientation Randomness Estimation: Fig. 18 shows the estimated degree of orientation randomness over the whole scene. As seen in Fig. 19, the SDEV of this parameter is very

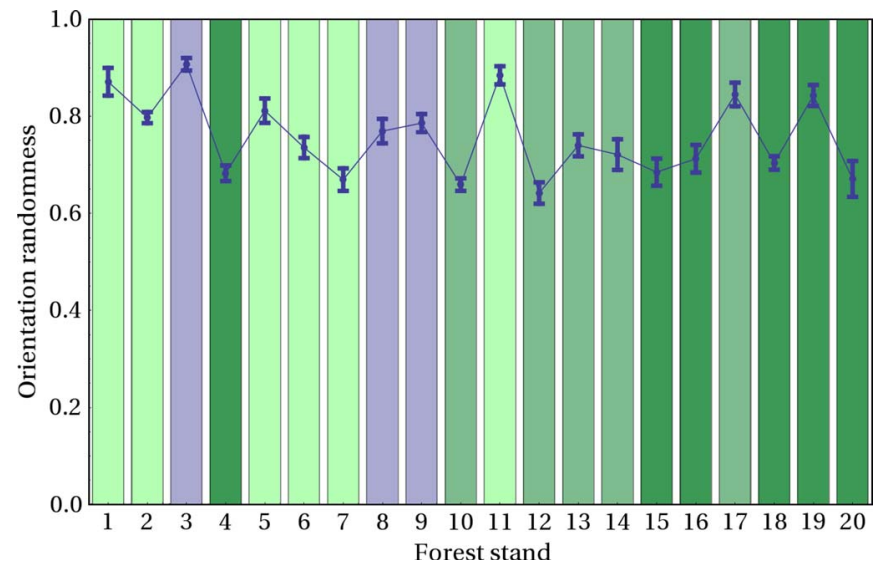

Fig. 19. Estimation of the degree of orientation randomness $\tau$. The error bars denote the estimated orientation randomness SDEV.

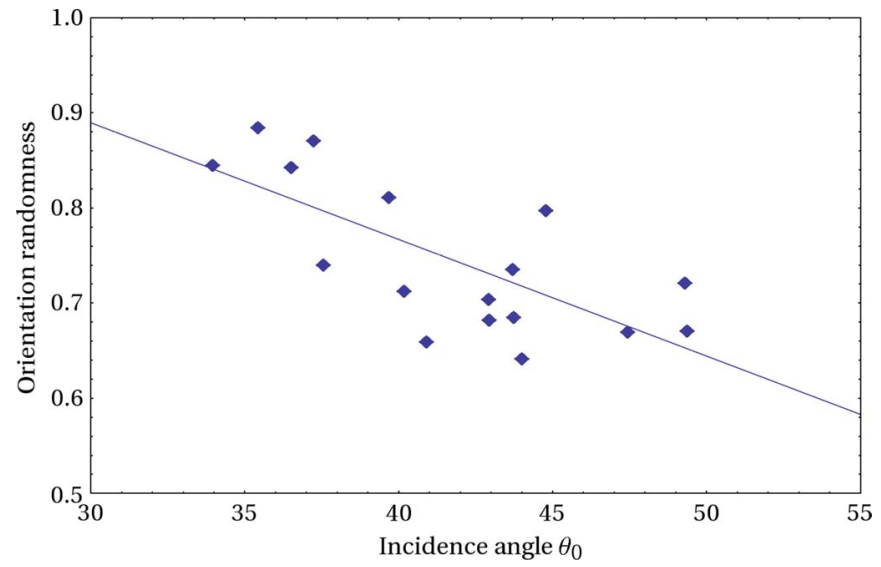

Fig. 20. Estimated degree of orientation randomness as a function of the angle of incidence (only for coniferous forest stands).

low, and the distinctiveness is high, which permits discriminating between the different evaluation stands. The background colors in this graph are related to dominant tree species type and growth stadium, with reference to Table II.

As discussed in the theoretical part, this parameter depends mostly on the morphology of the tree structures and the incidence angle. The incidence angle dependence is clearly observable in the scatter plot of $\tau$ over the incidence angles in Fig. 20.

5) Effective Particle Scattering Anisotropy Estimation: Surprisingly, the magnitude of the effective particle scattering anisotropy of the vegetation has been estimated to be larger than one, as shown in Fig. 21(a). The estimation is robust with very low variance (average SDEV is 0.035). Furthermore, it seems to be quite independent of the evaluation stand and the incidence angle. In contrast, the particle anisotropy phase [see Fig. 21(b)] has a large dynamic range of about $(2 / 5) \pi$ over the different evaluation stands. These observations need closer investigation.

The similarity of the particle scattering anisotropy magnitude over the evaluation stands might be explained with the fact that we observe relatively mixed forests consisting of several tree species in every evaluation stand. Given the different tree species in one sample, one has also to account the distribution of particle shapes, i.e., the range of branches from trunklike cylinders to medium-thickness branches to thin twigs. The 


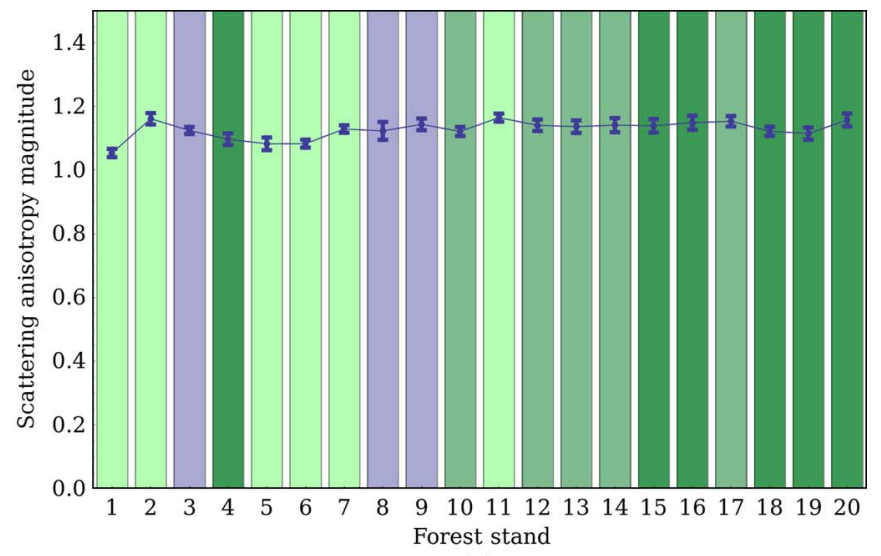

(a)

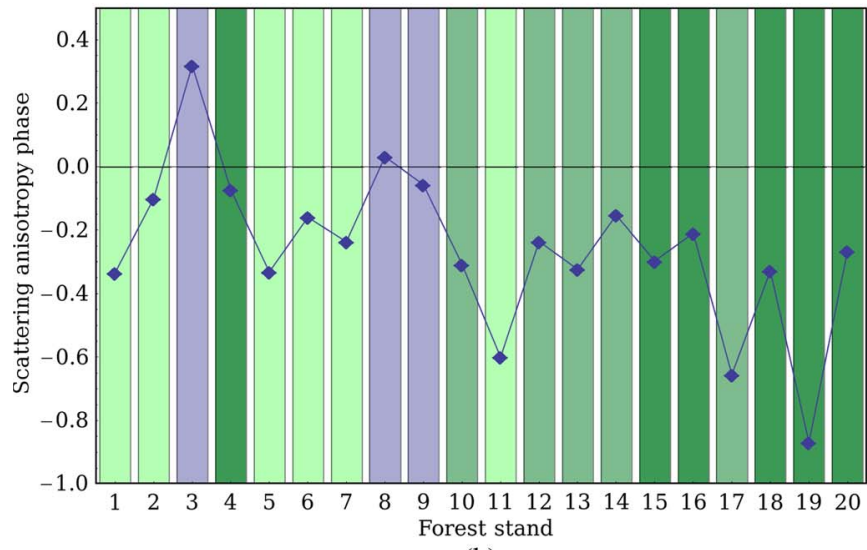

(b)

Fig. 21. Effective particle scattering anisotropy $\delta$. Magnitude and phase estimation. (a) Magnitude. (b) Phase.

distribution of the permittivities of the branches will probably vary slightly between the different tree species but not in a large extent. It is to expect that the permittivity will be much more affected by the time of the day/year and meteorological aspects than by the differences of given tree species mixes in the evaluation stands which grow under the same conditions. Therefore, it is to expect that one will obtain higher dynamical range of particle scattering anisotropy magnitudes for forests either under different meteorological conditions or at different times of the day or year; also, distinctively different plant morphologies (e.g., agricultural vegetation) should result in distinctive anisotropy magnitudes.

In theory, the particle scattering anisotropy magnitude under the Born approximation for a cloud of simple spheroidal particles is assumed to be restricted to the range of $[0,1]$. Exceeding of this range might have one of the following causes. First and most likely, it might be an indication for multiple scattering effects in the canopy which are neglected by the Born approximation but which could result in $|\delta|>1$. Second, it might be due to other unmodeled effects like a particular distribution of shape and dielectric properties of the scattering particles. Third, it might be due to the nonperfectly separated contributions from the ground. Fourth, miscalibration or processing artifacts can cause this behavior.

The phase of particle scattering anisotropy is more related to the orientation direction of the particles. The results in

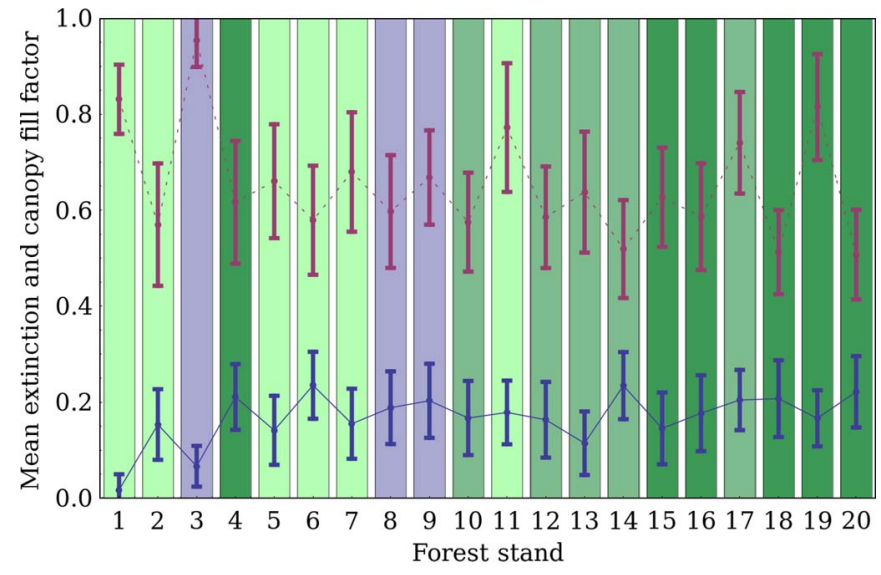

Fig. 22. Estimated mean and SDEV values of (solid blue line) extinction $\sigma$ and (dotted red line) canopy fill factor $r_{h}$.

Fig. 21(b) indicate an opportunity to discriminate forest tree species: Forest stands dominated by deciduous trees (stands 3 , 8, and 9; blue background) have positive (or very close to be positive in the case of stand 9) phases, whereas the stands dominated by coniferous trees (green background) have negative phases. Since this parameter is related to orientations, it is also sensitive to the incidence angle.

6) Extinction and Canopy Fill Factor Estimation: Extinction $\sigma$ and the canopy layer height ratio $r_{h}$ provide some degrees of freedom for the adaptation of the model to the given forest structure. However, together with the temporal decorrelation, these parameters are ambiguous to a certain degree. As can be seen in Fig. 22, the canopy fill factor is partly (negatively) correlated with the extinction. Both parameters are quite volatile in their ranges in the supposedly homogeneous evaluation stands. To validate the estimation of these parameters requires more information about the true structure of the analyzed forest stands, which is not available at the moment, thus making binding conclusions unfeasible.

7) Differential Extinction Estimation: Although not implemented in the presented parameter retrieval framework, one can reason about the value of the differential extinction [19], [20], [32] along the eigenpolarizations which causes polarization dependence of the volumetric coherence $\gamma_{z}$. Under the assumption that the extinction coefficient is proportional to the scattered intensity per unit volume, with respect to polarization change, one obtains the relationship

$$
\frac{\Delta \sigma}{\sigma} \approx\left|\frac{\mathrm{E}\left\{\left|S_{a a}\right|^{2}\right\}-\mathrm{E}\left\{\left|S_{b b}\right|^{2}\right\}}{\mathrm{E}\left\{\left|S_{a a}\right|^{2}\right\}+\mathrm{E}\left\{\left|S_{b b}\right|^{2}\right\}}\right| .
$$

Using the polarimetric relationship (37), one can obtain the estimates of the differential extinction $\Delta \sigma$ from the estimated values of $\delta, \tau$, and $\sigma$. The results are presented in Fig. 23, where the values of $\Delta \sigma$ range between 0 and $0.066 \mathrm{~dB} / \mathrm{m}$. Note that $\Delta \sigma$ scales mainly with $\sigma$ and $(1-\tau)$ and depends on the estimation accuracy of these values.

Introducing the estimated $\Delta \sigma$ into (25), together with the other estimated structure parameters, reveals that the variation in coherence magnitude due to the differential extinction $\left(\left|\gamma_{z(\sigma)}\right|-\left|\gamma_{z(\sigma \pm \Delta \sigma)}\right|\right)$ for the given data set is on the 


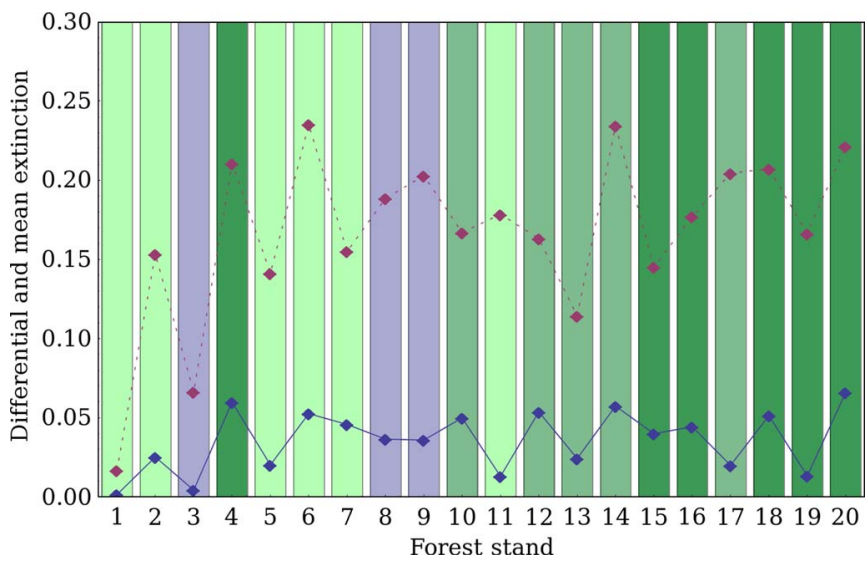

Fig. 23. (Solid blue line) Differential extinction $\Delta \sigma$, estimated from the (dotted red line) mean extinction $\sigma$ and the volume coherency matrix $\boldsymbol{T}_{v}$.

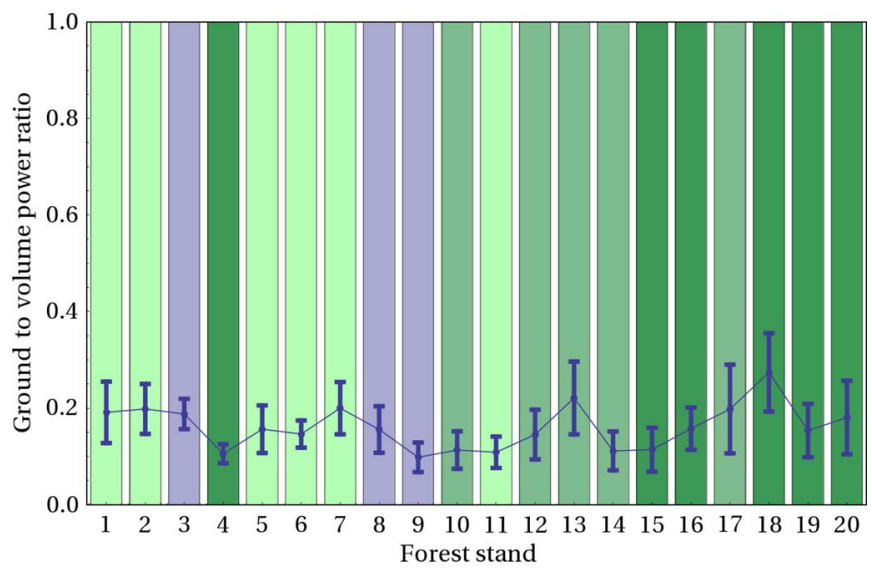

Fig. 24. Estimated ground-to-volume power ratio.

order of 0.001 [the mean (maximum in parentheses) values for baselines 1,2 , and 3 are $0.001(0.005), 0.005$ (0.018), and 0.0007 (0.0019), respectively]. In the coherence phase, the variation is on the order of $1^{\circ}$ [mean (maximum) values for all three baselines: $1.13^{\circ}\left(1.94^{\circ}\right), 2.12^{\circ}\left(3.66^{\circ}\right)$, and $\left.0.79^{\circ}\left(1.64^{\circ}\right)\right]$. The total difference between the complex coherence values $\left(\left|\gamma_{z(\sigma)}-\gamma_{z(\sigma \pm \Delta \sigma)}\right|\right)$ is on the order of 0.01 [mean (maximum) values: $0.018(0.03), 0.027(0.045)$, and $0.013(0.026)]$. These ranges are presumably below the noise level of the data.

8) Ground-to-Volume Power Ratio Estimation: Fig. 24 presents the estimated ground-to-volume power ratios $\left(P_{g} / P_{v}\right)$ for all forest stands, where

$$
P_{g}=f_{g} \operatorname{trace} \boldsymbol{T}_{g} \quad P_{v}=f_{v} \operatorname{trace} \boldsymbol{T}_{v} .
$$

This parameter is relatively volatile and has an SDEV of 0.1. The maximal power ratio is about three times higher than the minimal power ratio. No definitive conclusions about the relationships between this parameter and the tree species or the growth stadium or the height could be drawn up to now. An examination of the distinctively different forest types and/or different meteorological conditions could be advantageous for further analysis.

9) Ground Scattering Component Estimation: Fig. 25 shows the relative ground scattering components in the Pauli

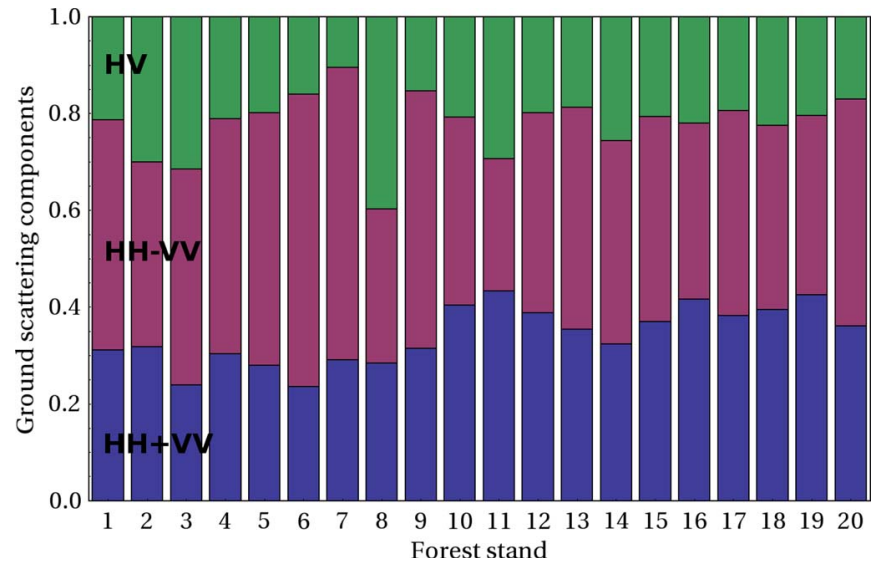

Fig. 25. Relative ground scattering components in the Pauli basis (from bottom to top). $H H+V V$ in blue, $H H-V V$ in red, and $\mathrm{HV}$ in green.

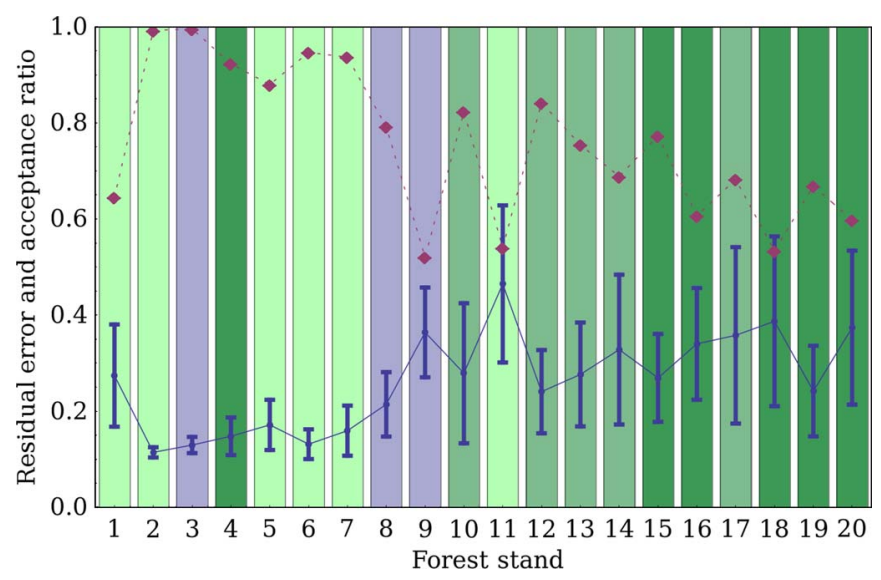

Fig. 26. (Solid blue line) Normalized residual error and (dotted red line) relative number of inverted pixels.

basis for all evaluation stands. Blue corresponds to $H H+V V$, red to $H H-V V$, and green to $H V$ intensities. Although the surface and double-bounce terms are large, the cross-polar component is significant, too. This is an additional indication that the simple first-order small perturbation model and the first-order double-bounce model are insufficient for modeling the ground contributions under the forest.

10) Inversion Performance and Residual Errors: Fig. 26 shows the average normalized residual errors and the relative number of inverted pixels (sample acceptance ratio). The criteria of acceptance were based on the residual error value and on the success of the inversion procedure to achieve physically reasonable results. This was not always the case due to several reasons. For instance, the inversion process was sometimes trapped in a local optimum or failed to converge, although a reasonable solution existed. It is also possible that the model used for inversion in these given pixels was inappropriate due to significant departure of the medium structure from the modeled medium.

It is expected that, by modification of the parameter estimation algorithm and by making it more robust, the performance could be further improved and the number of noninverted pixels could be further reduced. 
TABLE VII

HEIGHT ESTIMATION STATISTICS FOR DIFFERENT NUMBERS OF LOOKS

\begin{tabular}{c|ccc}
\hline \# of looks & Bias & RMSE & SDEV \\
\hline \hline 450 looks & -2.13 & 5.87 & 5.10 \\
900 looks & -1.75 & 5.61 & 4.88 \\
1350 looks & -1.60 & 5.43 & 4.77 \\
1800 looks & -1.51 & 4.97 & 4.33 \\
\hline
\end{tabular}

11) Number of Looks: Table VII presents the parameter estimation performance using different numbers of looks with respect to the retrieved forest height. These values have been computed independently for every forest stand, and, in the given table, the averages of these values over all stands are presented.

The results are according to the expectations: The forest height estimation errors, the height SDEV, and the residual errors are reduced with a higher number of looks. Reducing the number of looks by a factor of four (from 1800 to 450), the rmse is worsened by $15 \%-20 \%$. Over some evaluation stands (for instance, stands 2, 3, and 4), we always obtained good results, independent of the number of looks, which is an indication for the homogeneous structure of these stands. For low number of looks, we observed a trend for underestimation of high forest stands.

\section{CONCLUSION}

This paper has presented a model to characterize polarimetric interferometric radar response from vegetation. The model consists of volume and ground contributions for a PolInSAR repeat-pass configuration. For the volume component, a simple polarimetric model has been presented. Modeled as a cloud of discrete particles, it takes into account vegetation morphology in the form of effective particle scattering anisotropy and orientation distribution. The von Mises distribution has been recognized as the expected orientation angle distribution of vegetation particles, and a closed-form solution for the volume coherency matrix has been presented. The inverse problem of vegetation parameter retrieval from PolInSAR data is discussed, and a refined multistage procedure is developed to estimate all modeled parameters.

The limitations for the presented parameter retrieval framework are determined by the model assumptions. At first, the terrain slope is assumed to be constant over the region of the averaged samples. Only a simplified vegetation structure is taken into account, assuming vertically uniform and horizontally homogeneous layers. Next, it is assumed that the average effective particle shape is representable for all particles and independent of height and polarization orientation. The variation of extinction with polarization is assumed to be insignificant for parameter retrieval. Furthermore, a plane of reflection symmetry is assumed to exist for the illuminated vegetation area.

The parameter estimation performance has been evaluated on real airborne L-band SAR data in both SB and MB configurations. Using three baselines, the forest height has been underestimated with an average bias of $1.51 \mathrm{~m}$, an rmse of $4.97 \mathrm{~m}$, and an SDEV of $4.33 \mathrm{~m}$. In addition, one obtained the relatively

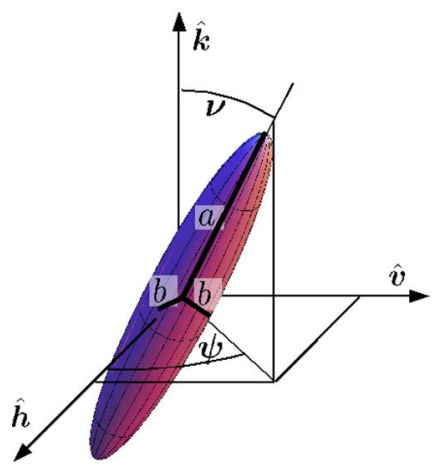

Fig. 27. Spheroidal particle dimensions and orientations in the antenna coordinate system. The azimuth angle $\psi$ (polarization orientation) is defined with respect to the $\hat{\boldsymbol{h}}$-axis and the zenith angle $\nu$ (tilt) with respect to the $\hat{\boldsymbol{k}}$-axis

reliable estimates of polarimetric indicators and backscattered powers for both layers, the ground and the canopy. On the other hand, the estimations of the extinction and the canopy fill factor were not satisfactory, as there seems to rest a level of ambiguity and correlation between these two parameters. This might be due to the strong simplification of the, in general quite complex, vertical structure of the forest. Furthermore, the differential extinction and the residual coherence, representing primarily the temporal decorrelation, have been estimated.

The presented model is quite flexible, permitting the construction of more complex multilayer models combining MB multitemporal multifrequency multiangular multialtitude measurements in a single parameter retrieval framework. The possibility to estimate the degree of temporal coherence over vegetation provides new opportunities for PolInSAR timeseries analysis, which might lead to competitive multitemporal monitoring of ecosystem dynamics. However, further theoretical and experimental investigations need to be conducted to improve the understanding and to examine the possibilities and limits of radar remote sensing of the temporal behavior and the forest structure.

\section{APPENDIX A}

\section{Relation of $\delta$ TO THE Spheroidal Particle Model}

The particle scattering anisotropy is a measure of the effective shape of the particle, as observed in the polarization plane. Using the spheroidal particle model from [2], [29], and [41], one can relate $\delta$ to real physical characteristics, assuming that the model corresponds to the illuminated particle.

Let the spheroidal particle be characterized by the halfaxes $a$ and $b$ and the polar angles $\psi$ and $\nu$ which describe the orientation and the tilt of the particle with respect to the LOS $(\hat{\boldsymbol{k}})$ and the polarization plane $(\hat{\boldsymbol{h}}-\hat{\boldsymbol{v}})$, as defined in Fig. 27. The polarizabilities $\alpha_{a}$ and $\alpha_{b}$ along the main axes are given by [41]

$$
\begin{aligned}
\alpha_{a} & =\frac{V}{4 \pi\left(L_{a}+1 /\left(\epsilon_{r}-1\right)\right)} \\
\alpha_{b} & =\frac{V}{4 \pi\left(L_{b}+1 /\left(\epsilon_{r}-1\right)\right)}
\end{aligned}
$$


where $V$ is the particle volume, $\epsilon_{r}$ is the permittivity, and $L_{a}$ and $L_{b}$ are defined as

$$
\begin{aligned}
L_{a} & =\int_{0}^{\infty} \frac{a b^{2}}{2\left(s+a^{2}\right)^{3 / 2}\left(s+b^{2}\right)} d s \\
L_{b} & =\int_{0}^{\infty} \frac{a b^{2}}{2\left(s+a^{2}\right)^{1 / 2}\left(s+b^{2}\right)^{2}} d s .
\end{aligned}
$$

$L_{a}$ and $L_{b}$ are related to particle dimensions via

$$
L_{a}+2 L_{b}=1
$$

and the aspect ratio $r$

$$
r=\frac{L_{a}}{L_{b}} \approx \frac{b}{a} \quad \begin{cases}r<1, & \text { prolate particles } \\ r=1, & \text { spherical particles } \\ r>1, & \text { oblate particles. }\end{cases}
$$

The backscattering matrix elements can be given in terms of particle polarizabilities and orientation angles by

$$
\begin{aligned}
& S_{h h}=\left(\alpha_{a}-\alpha_{b}\right) \sin ^{2} \nu \cos ^{2} \psi+\alpha_{b} \\
& S_{v v}=\left(\alpha_{a}-\alpha_{b}\right) \sin ^{2} \nu \sin ^{2} \psi+\alpha_{b} \\
& S_{h v}=\left(\alpha_{a}-\alpha_{b}\right) \sin ^{2} \nu \cos \psi \sin \psi .
\end{aligned}
$$

After the rotation of the polarization basis into the eigenbasis of the particle by (2), one obtains

$$
\boldsymbol{S}_{a b}=\left[\begin{array}{cc}
\alpha_{a} \sin ^{2} \nu+\alpha_{b} \cos ^{2} \nu & 0 \\
0 & \alpha_{b}
\end{array}\right] .
$$

Using the polarizability ratio $\alpha_{r}$, computed from (39)-(43) [2], [29]

$$
\alpha_{r}=\frac{\alpha_{a}}{\alpha_{b}}=\frac{r+\epsilon_{r}+1}{r \epsilon_{r}+2}
$$

the particle scattering anisotropy $\delta$ for a single spheroid can be given by

$$
\delta^{*}=\frac{S_{a a}-S_{b b}}{S_{a a}+S_{b b}}=\frac{\alpha_{r}-1}{\alpha_{r}+1+2 \cot ^{2} \nu} .
$$

Equation (49) provides the relationship between the particle scattering anisotropy and the single-particle characteristics.

For ensemble averages of spheroidal particles, (49) needs to be integrated over the distribution of the tilt angles $p_{\nu}$ as it has been done for the distribution of polarization orientation angles, assuming the independence of tilt angles from other particle characteristics. In particular, at an incidence angle of $45^{\circ}$, most media exhibit equal distributions for both $\psi$ and $\nu$. Assuming a circular normal distribution of the tilt angles as well, one can infer from $\delta$ the average polarizability ratio $\alpha$ of the volume particles for different degrees of orientation randomness $\tau$.

\section{APPENDIX B}

\section{InTERferometric Multilayer CoHerence Model}

In this appendix, the basic constituents of the interferometric coherence are derived based on a simplified model of SAR signals. The temporal and volume coherence constituents are further decomposed due to uncorrelated scattering contributions or multiple layers.

Let the SAR signal model be given under the plane wave approximation by [12], [13], [15]

$$
\begin{aligned}
s_{i}(x, R)=A e^{-2 i k_{0} R_{i 0}} \iiint & \hat{\boldsymbol{p}}_{r}^{\mathrm{T}} \boldsymbol{F}\left(\boldsymbol{r}^{\prime}\right) \hat{\boldsymbol{p}}_{t} e^{-2 i \boldsymbol{k} \cdot\left(\boldsymbol{r}^{\prime}-\boldsymbol{r}_{0}\right)} \\
& \times h\left(x-x^{\prime}, R-R^{\prime}\right) d V^{\prime}+n
\end{aligned}
$$

where $(x, R)$ are the SAR image azimuth and slant range coordinates, $\boldsymbol{k}=k_{0} \hat{\boldsymbol{k}}$ is the wave propagation vector propagating in the direction $\hat{k}$ with the wavenumber $k_{0}, R_{i 0}$ is the reference slant range distance, $\hat{\boldsymbol{p}}_{r}^{\mathrm{T}} \boldsymbol{F}\left(\boldsymbol{r}^{\prime}\right) \hat{\boldsymbol{p}}_{t}$ is the scattering amplitude matrix element projected to the antenna transmit and receive polarizations due to a scatterer at position $\boldsymbol{r}^{\prime}, h\left(x-x^{\prime}, R-R^{\prime}\right)$ is the SAR impulse response function, $n$ is the additive thermal noise, and $A$ represents the amplitude loss.

The cross correlation of two interferometric signals $s_{1}$ and $s_{2}$ can be given by

$$
\begin{aligned}
\mathrm{E}\left\{s_{1} s_{2}^{*}\right\}=A^{2} \iiint \mathrm{E}\left\{f_{1}(\boldsymbol{r}) f_{2}^{*}\left(\boldsymbol{r}^{\prime}\right)\right\}|h(\ldots)|^{2} \\
\times e^{2 i k_{0}\left(R_{2}^{\prime}-R_{1}^{\prime}\right)} d V^{\prime} .
\end{aligned}
$$

Let the scattering amplitudes $f\left(\boldsymbol{r}^{\prime}\right)=\hat{\boldsymbol{p}}_{r}^{\mathrm{T}} \boldsymbol{F}\left(\boldsymbol{r}^{\prime}\right) \hat{\boldsymbol{p}}_{t}$ follow the circular complex Gaussian distributions, and let the illuminated medium be horizontally homogeneous with a normalized distribution in height given by $\rho(z)\left(\int \rho(z) d z=1\right)$ so that the second-order scattering statistics can be described by

$$
\begin{aligned}
& \mathrm{E}\left\{f_{1}(\boldsymbol{r}) f_{1}^{*}\left(\boldsymbol{r}^{\prime}\right)\right\}=\mathrm{E}\left\{f_{2}(\boldsymbol{r}) f_{2}^{*}\left(\boldsymbol{r}^{\prime}\right)\right\}=\delta\left(\boldsymbol{r}-\boldsymbol{r}^{\prime}\right) \sigma_{v}^{0} \rho\left(z^{\prime}\right) \\
& \mathrm{E}\left\{f_{1}(\boldsymbol{r}) f_{2}^{*}\left(\boldsymbol{r}^{\prime}\right)\right\}=\delta\left(\boldsymbol{r}-\boldsymbol{r}^{\prime}\right) \sigma_{v e}^{0} \rho\left(z^{\prime}\right)
\end{aligned}
$$

where $\sigma_{v}^{0}$ is the total backscattering coefficient and $\sigma_{v e}^{0}$ is the temporally stable backscattering coefficient [12].

The Taylor series expansion of the phase delay difference provides [12], [13]

$$
2 i k_{0}\left(R_{2}-R_{1}\right) \approx i \phi_{0}+i k_{r}\left(R-R_{0}\right)+i k_{z}\left(z-z_{0}\right)
$$

where $k_{z}$ is given by (18), $k_{r}=2 k_{0}\left(B_{\perp} /\left(R_{0} \tan \theta_{0}\right)\right)$, and $\phi_{0}=2 k_{0}\left(R_{20}-R_{10}\right)$.

The auto- and cross correlations can thus be given by [12]-[15]

$$
\begin{aligned}
\mathrm{E}\left\{s_{1} s_{2}^{*}\right\}= & A^{2} e^{i \phi_{0}} \sigma_{v e}^{0} \iint|h(\ldots)|^{2} e^{i k_{r}\left(R^{\prime}-R_{0}\right)} d R^{\prime} d x^{\prime} \\
& \times \int \rho\left(z^{\prime}\right) e^{i k_{z}\left(z^{\prime}-z_{0}\right)} d z^{\prime} \\
= & A^{2} e^{i \phi_{0}} \sigma_{v e}^{0} I_{r x} I_{z} \\
\mathrm{E}\left\{s_{1} s_{1}^{*}\right\}= & \mathrm{E}\left\{s_{2} s_{2}^{*}\right\} \\
= & A^{2} \sigma_{v}^{0} \iint|h(\ldots)|^{2} d R^{\prime} d x^{\prime} \int \rho\left(z^{\prime}\right) d z^{\prime}+\sigma_{n} \\
= & A^{2} \sigma_{v}^{0} I_{r x}^{0} I_{z}^{0}+\sigma_{n}
\end{aligned}
$$


where $\sigma_{n}=\mathrm{E}\left\{\left|n^{2}\right|\right\}$ is the noise variance and $I_{r x}, I_{z}, I_{r x}^{0}$, and $I_{z}^{0}$ represent the corresponding integrals over the range and azimuth and over the height dimensions.

The complex coherence for this layer can thus be given by

$$
\begin{aligned}
\gamma & =\frac{\mathrm{E}\left\{s_{1} s_{2}^{*}\right\}}{\sqrt{\mathrm{E}\left\{\left|s_{1}\right|^{2}\right\} \mathrm{E}\left\{\left|s_{2}\right|^{2}\right\}}} \\
& =e^{i \phi_{0}} \frac{\sigma_{v e}^{0}}{\sigma_{v}^{0}} \frac{I_{z}}{I_{z}^{0}} \frac{I_{r x}}{I_{r x}^{0}} \frac{\sigma_{0}}{\sigma_{0}+\sigma_{n}} \\
& =e^{i \phi_{0}} \gamma_{\text {temp }} \gamma_{z} \gamma_{r} \gamma_{\text {therm }}
\end{aligned}
$$

where $\sigma_{0}=A^{2} \sigma_{v}^{0} I_{r x}^{0} I_{z}^{0}$ is the noise-free autocorrelation and $\gamma_{\text {temp }}, \gamma_{z}, \gamma_{r}$, and $\gamma_{\text {therm }}$ represent the temporal, volume, range, and thermal decorrelation sources, respectively.

Having an attenuating medium with extinction coefficient $\sigma$ and the normalized scattering height profile distribution

$$
\rho(z)=\frac{e^{\frac{2 \sigma}{\cos \theta_{0}} z}}{\int e^{\frac{2 \sigma}{\cos \theta_{0}} z^{\prime}} d z^{\prime}}
$$

leads to the volume coherence expression in (25). In (23) and (24), the range decorrelation $\gamma_{r}$ has been compensated by the wavenumber shift [42], and $\gamma_{\text {sys }}$ consists of $\gamma_{\text {therm }}$, as well as other possible sources of decorrelation which are not related to volume and temporal coherences $\gamma_{z}$ and $\gamma_{\text {temp }}$.

Having two uncorrelated and/or separated layers with the following scattering properties

$$
\begin{aligned}
& \mathrm{E}\left\{f_{1}(\boldsymbol{r}) f_{1}^{*}\left(\boldsymbol{r}^{\prime}\right)\right\}=\delta\left(\boldsymbol{r}-\boldsymbol{r}^{\prime}\right)\left(\sigma_{v 1}^{0} \rho_{1}\left(z^{\prime}\right)+\sigma_{v 2}^{0} \rho_{2}\left(z^{\prime}\right)\right) \\
& \mathrm{E}\left\{f_{1}(\boldsymbol{r}) f_{2}^{*}\left(\boldsymbol{r}^{\prime}\right)\right\}=\delta\left(\boldsymbol{r}-\boldsymbol{r}^{\prime}\right)\left(\sigma_{v 2 e}^{0} \rho_{2}\left(z^{\prime}\right)+\sigma_{v 2 e}^{0} \rho_{2}\left(z^{\prime}\right)\right)
\end{aligned}
$$

the auto- and cross correlations can be recomputed to provide the complex coherence expression

$$
\gamma=e^{i \phi_{0}} \frac{I_{r x}}{I_{r x}^{0}}\left(\frac{\sigma_{v e 1}^{0} I_{z 1}+\sigma_{v e 2}^{0} I_{z 2}}{\sigma_{v 1}^{0} I_{z 1}^{0}+\sigma_{v 2}^{0} I_{z 2}^{0}}\right) \frac{\sigma_{0}}{\sigma_{0}+\sigma_{n}}
$$

where $\sigma_{0}=A^{2} I_{r x}^{0}\left(\sigma_{v 1}^{0} I_{z 1}^{0}+\sigma_{v 2}^{0} I_{z 2}^{0}\right)$.

Using the intensity ratio [20]

$$
\Delta=\frac{\sigma_{v 1}^{0} I_{z 1}^{0}}{\sigma_{v 2}^{0} I_{z 2}^{0}}
$$

or the normalized intensity weights

$$
\begin{aligned}
& c_{1}=\frac{\sigma_{v 1}^{0} I_{z 1}^{0}}{\sigma_{v 1}^{0} I_{z 1}^{0}+\sigma_{v 2}^{0} I_{z 2}^{0}}=\frac{\Delta}{\Delta+1} \\
& c_{2}=\frac{\sigma_{v 2}^{0} I_{z 2}^{0}}{\sigma_{v 1}^{0} I_{z 1}^{0}+\sigma_{v 2}^{0} I_{z 2}^{0}}=\frac{1}{\Delta+1}
\end{aligned}
$$

the coherence for two uncorrelated layers can be expressed by

$$
\begin{aligned}
\gamma & =e^{i \phi_{0}} \gamma_{r}\left(\frac{\Delta \gamma_{\text {temp } 1} \gamma_{z 1}+\gamma_{\text {temp } 2} \gamma_{z 2}}{\Delta+1}\right) \gamma_{\text {therm }} \\
& =e^{i \phi_{0}} \gamma_{r}\left(c_{1} \gamma_{\text {temp } 1} \gamma_{z 1}+c_{2} \gamma_{\text {temp } 2} \gamma_{z 2}\right) \gamma_{\text {therm }} .
\end{aligned}
$$

This two-layer structure can readily be generalized to multiple layers

$$
\begin{aligned}
\gamma & =e^{i \phi_{0}} \gamma_{r}\left(\frac{\sum_{i=1}^{N} \Delta_{i}^{1} \gamma_{\text {temp }, i} \gamma_{z, i}}{\sum_{i=1}^{N} \Delta_{i}^{1}}\right) \gamma_{\text {therm }} \\
& =e^{i \phi_{0}} \gamma_{r}\left(\sum_{i=1}^{N} c_{i} \gamma_{\text {temp }, i} \gamma_{z, i}\right) \gamma_{\text {therm }}
\end{aligned}
$$

where $\Delta_{i}^{1}$ is the intensity ratio of layer 1 to layer $i$ and $c_{i}$ is the normalized intensity weight of layer $i\left(\sum c_{i}=1\right)$.

\section{ACKNOWLEDGMENT}

The authors would like to thank both reviewers for their perceptive comments and valuable recommendations with respect to this paper.

\section{REFERENCES}

[1] S. R. Cloude and E. Pottier, "An entropy based classification scheme for land applications of polarimetric SAR," IEEE Trans. Geosci. Remote Sens., vol. 35, no. 1, pp. 68-78, Jan. 1997.

[2] S. R. Cloude, J. Fortuny, J. M. Lopez-Sanchez, and A. J. Sieber, "Wideband polarimetric radar inversion studies for vegetation layers," IEEE Trans. Geosci. Remote Sens., vol. 37, no. 5, pp. 2430-2441, Sep. 1999.

[3] D. L. Schuler, J. S. Lee, D. Kasilingam, and G. Nesti, "Surface roughness and slope measurements using polarimetric SAR data," IEEE Trans. Geosci. Remote Sens., vol. 40, no. 3, pp. 687-698, Mar. 2002.

[4] J. S. Lee, D. L. Schuler, T. L. Ainsowrth, E. Krogager, D. Kasilingam, and W. M. Boerner, "On the estimation of radar polarization orientation shifts induced by terrain slopes," IEEE Trans. Geosci. Remote Sens., vol. 40, no. 1, pp. 30-41, Jan. 2002.

[5] J. van Zyl, "Unsupervised classification of scattering behavior using radar polarimetry data," IEEE Trans. Geosci. Remote Sens., vol. 27, no. 1, pp. 36-45, Jan. 1989.

[6] S. R. Cloude and E. Pottier, "A review of target decomposition theorems in radar polarimetry," IEEE Trans. Geosci. Remote Sens., vol. 34, no. 2, pp. 498-518, Mar. 1996.

[7] A. Freeman and S. L. Durden, "A three-component model for polarimetric SAR imagery," IEEE Trans. Geosci. Remote Sens., vol. 34, no. 3, pp. 963-973, May 1998.

[8] A. Freeman, "Fitting a two-component scattering model to polarimetric SAR data from forests," IEEE Trans. Geosci. Remote Sens., vol. 45, no. 8, pp. 2583-2592, Aug. 2007.

[9] Y. Yamaguchi, T. Moriyama, M. Ishido, and H. Yamada, "Fourcomponent scattering model for polarimetric SAR image decomposition," IEEE Trans. Geosci. Remote Sens., vol. 43, no. 8, pp. 1699-1706, Aug. 2005.

[10] Y. Yamaguchi, Y. Yajima, and H. Yamada, "A four-component decomposition of POLSAR images based on the coherency matrix," IEEE Geosci. Remote Sens. Lett., vol. 3, no. 3, pp. 292-296, Jul. 2006.

[11] E. Rodriguez and J. M. Martin, "Theory and design of interferometric synthetic aperture radars," Proc. Inst. Elect. Eng., vol. 139, no. 2, pp. 147-159, Apr. 1992.

[12] J. O. Hagberg, L. M. H. Ulander, and J. Askne, "Repeat-pass SAR interferometry over forested terrain," IEEE Trans. Geosci. Remote Sens., vol. 33, no. 2, pp. 331-340, Mar. 1995.

[13] R. N. Treuhaft, S. N. Madsen, M. Moghaddam, and J. J. van Zyl, "Vegetation characteristics and underlying topography from interferometric radar," Radio Sci., vol. 31, no. 6, pp. 1449-1485, Nov. 1996.

[14] J. I. H. Askne, P. B. G. Dammert, L. M. H. Ulander, and G. Smith, "C-band repeat-pass interferometric SAR observations of the forest," IEEE Trans. Geosci. Remote Sens., vol. 35, no. 1, pp. 25-35, Jan. 1997.

[15] R. Bamler and P. Hartl, "Synthetic aperture radar interferometry," Inv. Probl., vol. 14, no. 4, pp. R1-R54, Aug. 1998.

[16] P. A. Rosen, S. Hensley, I. R. Joughin, F. K. Li, S. N. Madsen, E. Rodriguez, and R. M. Goldstein, "Synthetic aperture radar interferometry," Proc. IEEE, vol. 88, no. 3, pp. 333-382, Mar. 2000. 
[17] M. Santoro, J. Askne, and P. B. G. Dammert, "Tree height influence on ERS interferometric phase in Boreal forest," IEEE Trans. Geosci. Remote Sens., vol. 43, no. 2, pp. 207-217, Feb. 2005.

[18] S. R. Cloude and K. P. Papathanassiou, "Polarimetric SAR interferometry," IEEE Trans. Geosci. Remote Sens., vol. 36, no. 5, pp. 1551-1565, Sep. 1998.

[19] R. N. Treuhaft and S. R. Cloude, "The structure of oriented vegetation from polarimetric interferometry," IEEE Trans. Geosci. Remote Sens., vol. 37, no. 5, pp. 2620-2624, Sep. 1999.

[20] R. N. Treuhaft and P. R. Siqueira, "Vertical structure of vegetated land surfaces from interferometric and polarimetric radar," Radio Sci., vol. 35, no. 1, pp. 141-178, Jan. 2000.

[21] K. P. Papathanassiou and S. R. Cloude, "Single-baseline polarimetric SAR interferometry," IEEE Trans. Geosci. Remote Sens., vol. 39, no. 11, pp. 2352-2363, Nov. 2001.

[22] S. R. Cloude and K. P. Papathanassiou, "Three-stage inversion process for polarimetric SAR interferometry," Proc. Inst. Elect. Eng.-Radar Sonar, Navig., vol. 150, no. 3, pp. 125-134, Jun. 2003.

[23] H. Yamada, Y. Yamaguchi, Y. Kim, E. Rodriguez, and W. M. Boerner, "Polarimetric SAR interferometry for forest analysis based on the ESPRIT algorithm," IEICE Trans. Electron., vol. E84-C, no. 12, pp. 1917-1924, Dec. 2001.

[24] J. D. Ballester-Berman, J. M. Lopez-Sanchez, and J. Fortuny-Guasch, "Retrieval of biophysical parameters of agricultural crops using polarimetric SAR interferometry," IEEE Trans. Geosci. Remote Sens., vol. 43, no. 4, pp. 683-694, Apr. 2005.

[25] J. Praks, F. Kugler, K. P. Papathanassiou, I. Hajnsek, and M. Hallikainen, "Height estimation of boreal forest: Interferometric model-based inversion at L- and X-band versus HUTSCAT profiling scatterometer," IEEE Geosci. Remote Sens. Lett., vol. 4, no. 3, pp. 466-470, Jul. 2007.

[26] S. K. Lee, F. Kugler, K. Papathanassiou, and I. Hajnsek, "Quantifying temporal decorrelation over boreal forest at L- and P-band," in Proc. EUSAR, Friedrichshafen, Germany, Jun. 2008.

[27] F. Garestier, P. C. Dubois-Fernandez, and I. Champion, "Forest height inversion using high-resolution P-band Pol-InSAR data," IEEE Trans. Geosci. Remote Sens., vol. 46, no. 11, pp. 3544-3599, Nov. 2008.

[28] M. Neumann, L. Ferro-Famil, and A. Reigber, "Modeling and interpretation of the multitemporal and multibaseline PolInSAR coherence," in Proc. IEEE IGARSS, Boston, MA, Jul. 2008, pp. II-477-II-480.

[29] B. P. Ablitt, "Characterisation of particles and their scattering effects on polarized light," Ph.D. dissertation, Univ. Nottingham, Nottingham, U.K., Mar. 2000.

[30] M. Abramowitz and I. A. Stegun, Handbook of Mathematical Functions with Formulas, Graphs, and Mathematical Tables. New York: Dover, 1964.

[31] L. Ferro-Famil and M. Neumann, "Recent advances in the derivation of Pol-InSAR statistics: Study and applications," in Proc. EUSAR, Friedrichshafen, Germany, Jun. 2008

[32] M. Neumann, "Remote sensing of vegetation using multi-baseline polarimetric SAR interferometry: Theoretical modeling and physical parameter retrieval," Ph.D. dissertation, Univ. Rennes 1, Rennes, France, Jan. 2009.

[33] K. P. Papathanassiou, S. R. Cloude, A. Reigber, and W. M. Boerner, "Multi-baseline polarimetric SAR interferometry for vegetation parameter estimation," in Proc. IEEE IGARSS, Honolulu, HI, Jul. 2000, vol. 6, pp. 2762-2764.

[34] R. Snieder, "The role of nonlinearity in inverse problems," Inv. Probl., vol. 14, no. 3, pp. 387-404, Jun. 1998.

[35] M. Moghaddam, "Effect of medium symmetries in limiting the number of parameters estimated with polarimetric interferometry," in Proc. IEEE IGARSS, Hamburg, Germany, 1999, pp. 2221-2223.

[36] R. Touzi, A. Lopes, J. Bruniquel, and P. W. Vachon, "Coherence estimation for SAR imagery," IEEE Trans. Geosci. Remote Sens., vol. 37, no. 1, pp. 135-149, Jan. 1999.

[37] W. H. Press, S. A. Teukolsky, W. T. Vetterling, and B. P. Flannery, Numerical Recipes in C: The Art of Scientific Computing. Cambridge, U.K.: Cambridge Univ. Press, 1992.

[38] T. Mette, "Forest biomass estimation from polarimetric SAR interferometry," Ph.D. dissertation, Tech. Univ. München, München, Germany, 2007.

[39] H. A. Zebker and J. Villasenor, "Decorrelation in interferometric radar echoes," IEEE Trans. Geosci. Remote Sens., vol. 30, no. 5, pp. 950-959, Sep. 1992.
[40] F. Rocca, "Modeling interferogram stacks," IEEE Trans. Geosci. Remote Sens., vol. 45, no. 10, pp. 3289-3299, Oct. 2007.

[41] H. C. van de Hulst, Light Scattering by Small Particles. New York: Dover, 1981

[42] F. Gatelli, A. M. Guarnieri, F. Parizzi, P. Pasquali, C. Prati, and F. Rocca, "The wavenumber shift in SAR interferometry," IEEE Trans. Geosci. Remote Sens., vol. 32, no. 4, pp. 855-865, Jul. 1994.

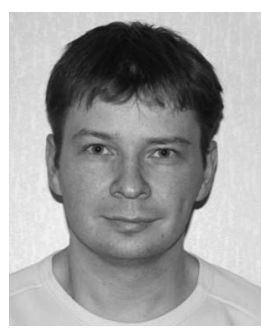

Maxim Neumann (S'06) was born in Dsheskasgan, Kazakhstan. He received the Dipl.Ing. degree (with distinction) in computer engineering from the Berlin University of Technology, Berlin, Germany, in 2004 and the Ph.D. degree from the University of Rennes 1, Rennes, France, in 2009.

From 2002 to 2003, he was with the Media and Machines Laboratory, Washington University in St. Louis, St. Louis, MO, where he worked on machine learning methods for mobile robots. From 2004 to 2005, he was with the Computer Vision and Remote Sensing Group, Berlin University of Technology, Berlin. Since 2006, he is with the SAPHIR Team, Institute of Electronics and Telecommunications of Rennes, University of Rennes 1 . His current research interests include SAR polarimetry and interferometry with respect to electromagnetic scattering modeling and vegetation parameter retrieval.

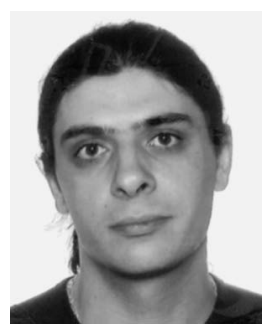

Laurent Ferro-Famil (S'99-A'00-M'04) received the Laurea degree in electronics systems and computer engineering, the M.S. degree in electronics, and the Ph.D. degree from the University of Nantes, Nantes, France, in 1996, 1996, and 2000, respectively.

In 2001, he became an Assistant Professor, and, since 2007, he has been an Associate Professor with the University of Rennes 1, Rennes, France, where he is currently a Member of the Radar Polarimetry Remote Sensing Group, Institute of Electronics and Telecommunications of Rennes. His current activities in education are concerned with analog electronics, digital communications, microwave theory, and polarimetric SAR remote sensing. He is particularly interested in polarimetric SAR signal processing, radar polarimetry theory, and natural media remote sensing from multibaseline PolInSAR data, with application to classification, electromagnetic scattering modeling and physical parameter inversion, time-frequency analysis, and 3-D reconstruction of environments.

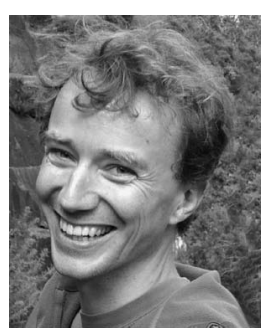

Andreas Reigber (M'02) was born in Munich, Germany, in 1970. He received the diploma degree in physics from the University of Konstanz, Konstanz, Germany, in 1997, the Ph.D. degree from the University of Stuttgart, Stuttgart, Germany, in 2001, and the Habilitation from the Berlin University of Technology, Berlin, Germany, in 2008.

From 1996 to 2000, he was with the Microwaves and Radar Institute, German Aerospace Center (DLR), Wessling, Germany, where he worked in the field of polarimetric SAR tomography. In 2001, he joined the Antenna, Radar and Telecom Laboratories, University of Rennes 1, Rennes, France, for postdoctoral research on radar polarimetry and polarimetric interferometry. From 2002 to 2007, he was a Research Associate with the Computer Vision and Remote Sensing Laboratories, Berlin University of Technology. Since 2008, he has been with the DLR Microwaves and Radar Institute, where he is currently the Head of the SAR Technology Department and directing the airborne SAR activities of the institute. His current main research interests are not only the various aspects of multimodal SAR, like SAR interferometry, SAR polarimetry, SAR tomography, and time-frequency analyses, but also the application of computer vision and machine learning approaches in remote sensing. 